\title{
Data Quality and Cost-effectiveness Analyses of Electronic and Paper-Based Interviewer-Administered Public Health Surveys: Systematic Review
}

Atinkut Alamirrew Zeleke ${ }^{1,2}, \mathrm{PhD}$; Tolga Naziyok ${ }^{2}$, MSc; Fleur Fritz ${ }^{3}, \mathrm{PhD}$; Lara Christianson ${ }^{4}$, MLS; Rainer Röhrig ${ }^{2,5}$, MD

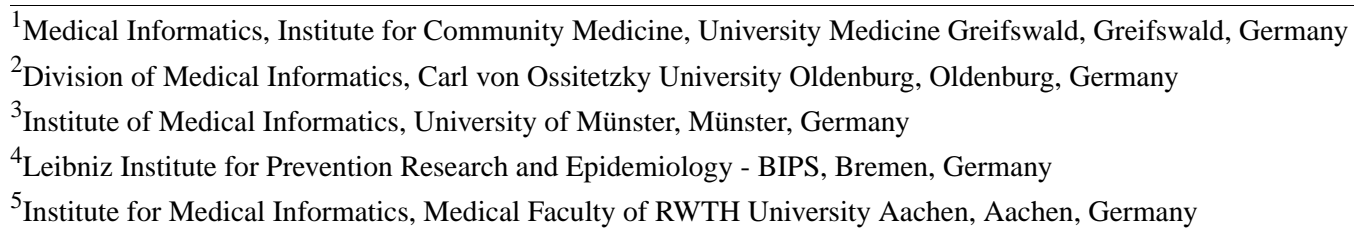

Corresponding Author:

Atinkut Alamirrew Zeleke, PhD

Medical Informatics

Institute for Community Medicine

University Medicine Greifswald

Ellernholzstraße 1-2

Greifswald, D-17487

Germany

Phone: 493834867548

Email: atinkut.zeleke@uni-greifswald.de

\section{Abstract}

Background: A population-level survey (PLS) is an essential and standard method used in public health research that supports the quantification of sociodemographic events, public health policy development, and intervention designs. Data collection mechanisms in PLS seem to be a significant determinant in avoiding mistakes. Using electronic devices such as smartphones and tablet computers improves the quality and cost-effectiveness of public health surveys. However, there is a lack of systematic evidence to show the potential impact of electronic data collection tools on data quality and cost reduction in interviewer-administered surveys compared with the standard paper-based data collection system.

Objective: This systematic review aims to evaluate the impact of the interviewer-administered electronic data collection methods on data quality and cost reduction in PLS compared with traditional methods.

Methods: We conducted a systematic search of MEDLINE, CINAHL, PsycINFO, the Web of Science, EconLit, Cochrane CENTRAL, and CDSR to identify relevant studies from 2008 to 2018 . We included randomized and nonrandomized studies that examined data quality and cost reduction outcomes, as well as usability, user experience, and usage parameters. In total, 2 independent authors screened the title and abstract, and extracted data from selected papers. A third author mediated any disagreements. The review authors used EndNote for deduplication and Rayyan for screening.

Results: Our search produced 3817 papers. After deduplication, we screened 2533 papers, and 14 fulfilled the inclusion criteria. None of the studies were randomized controlled trials; most had a quasi-experimental design, for example, comparative experimental evaluation studies nested on other ongoing cross-sectional surveys. A total of 4 comparative evaluations, 2 pre-post intervention comparative evaluations, 2 retrospective comparative evaluations, and 4 one-arm noncomparative studies were included. Meta-analysis was not possible because of the heterogeneity in study designs, types, study settings, and level of outcome measurements. Individual paper synthesis showed that electronic data collection systems provided good quality data and delivered faster compared with paper-based data collection systems. Only 2 studies linked cost and data quality outcomes to describe the cost-effectiveness of electronic data collection systems. Field data collectors reported that an electronic data collection system was a feasible, acceptable, and preferable tool for their work. Onsite data error prevention, fast data submission, and easy-to-handle devices were the comparative advantages offered by electronic data collection systems. Challenges during implementation included technical difficulties, accidental data loss, device theft, security concerns, power surges, and internet connection problems. 
Conclusions: Although evidence exists of the comparative advantages of electronic data collection compared with paper-based methods, the included studies were not methodologically rigorous enough to combine. More rigorous studies are needed to compare paper and electronic data collection systems in public health surveys considering data quality, work efficiency, and cost reduction.

\section{International Registered Report Identifier (IRRID)： RR2-10.2196/10678}

(J Med Internet Res 2021;23(1):e21382) doi: 10.2196/21382

\section{KEYWORDS}

electronic data collection; demographic and health survey; tablet computer; smartphone; mobile phone

\section{Introduction}

Until well-established civil and vital statistics systems are in place in low- and middle-income countries (LMIC), monitoring sociodemographic events using data on vital societal statistics will remain dependent on alternative data sources. Public health surveys - such as censuses, demographic and health surveys (DHS), and health and demographic surveillance-serve as a data lifeline for these countries [1,2]. Mortality and morbidity indicators, service utilization, and population-level program impact evaluations are usually calculated from household-level data. Further analysis of these population-level epidemiologic indicators is helpful in identifying the determinants of mortality and morbidity. Data collection and management is the first step in the process of evidence generation from household surveys, in which data quality errors could be introduced or prevented. Avoiding errors at this stage is the first-line choice to avoid inherited errors in further data management processes $[1,3,4]$.

The current classical data collection and management processes in LMIC are heavily dependent on paper-based manual methods $[4,5]$. Paper-based data collection requires extensive human and material resources, especially for large-scale surveys. It also incurs high printing and data entry costs and requires extra data quality assurance steps during and after data collection. Moreover, it takes a long time for an error-free data set to be ready for analysis $[6,7]$. The intrinsic mode of paper-based data collection affects the data quality, timeliness, and cost of survey implementation, among other factors [8-10].

The rapid development of the global telecommunications infrastructure provides an opportunity for mobile and wireless technologies (mobile health [mHealth]) to support health services and research. Harnessing this technology's potential, particularly in LMIC where the disease burden is highest, is becoming a popular strategy led by relevant activities in World Health Organization member countries [11]. There are diverse mHealth solutions broadly categorized as a tool to support communication between health service institutions and individuals. These include health call centers; reminders to attend appointments; providing access to information and education for health care professionals, for example, access to electronic health care databases and clinical decision support systems; and supporting health monitoring and surveillance (eg, data collection and reporting in health surveys, surveillance, and patient monitoring) [12]

The implementation of tablet- or smartphone-based data collection tools is becoming increasingly popular in public health surveys to mitigate challenges encountered in paper-based data collection $[13,14]$. Compared with face-to-face interviews, a self-administering mode of electronic data collection tools could potentially increase the response rate among stigmatized groups. These tools have been tested in the contexts of drug abuse [15] and sexual health or HIV [16-18] in public health. The findings conclude that respondents prefer electronic data collection tools as a solution for reporting sensitive information.

Considering data collection in clinical trials, electronic clinical report forms (eCRF) show a potential advantage over paper-based clinical case report forms (CRF) [19-22]. Studies have identified the relative advantages of electronic data capturing tools in terms of data quality, timeliness, and implementation cost.

A handful of experience reports are available on the use of electronic data collection methods in health and demographic surveillance systems (HDSS) in the International Network for the Demographic Evaluation of Populations and Their Health (INDEPTH) network. The HDSS site in Malawi used an OpenHDS data system as a means of GPS data collection [23]. One surveillance site in Kenya also reported the adoption of technological innovation using OpenHDS to manage a large-scale malaria survey in western Kenya. The findings asserted that electronic data collection (EDC) enabled the collection of demographic and malaria data quickly and effectively. Moreover, the possibility of real-time data quality controls using the system led to an efficient workflow and subsequent cost savings [24]. The Kombewa HDSS in Kenya also collected data electronically using PDAs and computer notebooks [25,26]. Since 2010, the Magu HDSS site in Tanzania has used EDC to enable enumerators to record household information directly in the PDA [27]. The Dabat HDSS site in northwest Ethiopia also reported the use of PDAs as a means of data collection [6,28]. Most HDSS and DHS still use a paper-based data collection system, and those sites with EDC implementation experience have rarely published their experience or the comparative impact of EDC and paper-based data collections. Despite the individual implementation experiences that suggest that EDC tools can improve data quality and work efficiency and reduce overall survey costs, systematic reviews of the available evidence are limited. The focus of the available systematic reviews is primarily on the mixed potential of mHealth, not specifically on the impact of mobile devices on improving the data collection and management processes in surveys $[13,29,30]$. Therefore, the impacts of EDC tools in surveys need to be separately analyzed and reported. The available Cochrane systematic review on the impact of data 
quality parameters focuses on self-administered EDC tools and excludes interviewer-administered methods [14]. In the case of face-to-face interviews, the data collection process involves interaction between the questionnaire, respondent, and interviewer. The difference in the mode of questionnaire administration can have serious effects on data quality [9]. Moreover, conducting face-to-face surveys has more organizational costs involved than self-administered surveys.

Therefore, a systematic review that considers interviewer-administered data collection may complement this evidence. We found no systematic review that analyzed the data quality and cost-effectiveness of electronic and paper-based interview-administered public health surveys. The objective of this systematic review is to synthesize the evidence on the effect of using EDC systems on data quality and cost reduction in public health surveys, with a focus on studies reporting comparative impacts of paper-based data collection and EDC.

\section{Methods}

We registered a detailed protocol with PROSPERO, an international database of prospectively registered systematic reviews, with the registration number CRD42018092259. PRISMA (Preferred Reporting Items for Systematic Review and Meta-Analysis) guidelines were used to report our systematic review [31,32]. The protocol of this study has been published [33].

\section{Inclusion Criteria}

We assessed studies that investigated the effect of EDC methods on improving the data quality and cost-effectiveness in public health surveys or surveillance, compared with traditional paper-based data collection methods. We included all mobile apps with technologies that directly support the data collection process by enabling data collectors and interviewers to collect and send data as well as enabling supervisors and data managers to monitor the data collection process. The study participants included in our review are defined as data collection tool users who use a method of data collection.

Studies with the following characteristics were included:

- The study compared either data quality or cost-effectiveness or both as primary outcomes and reported these in the paper.

- The intervention consisted of mobile information and communication technology devices along with mobile apps, which include PDAs, cellphones, smartphones, and tablet computers-devices used specifically for data collection and reporting processes during surveys.

- The control and intervention groups were compared in face-to-face interview-administered surveys conducted at the household level.

- Demographic surveillance sites were based on clinical settings and not mandated for standard clinical trials (eg, CRF vs eCRF).

- The paper was published between January 2008 and December 2018.

\section{Search Information Source and Search Strategies}

Studies were identified through systematic searching in the following electronic databases: MEDLINE via Ovid, CINAHL via EBSCO, PsycINFO via Ovid, EconLit via EBSCO, the Social Science Citation Index, the Science Citation Index via the Web of Science and CENTRAL, and the Cochrane Library (Table 1). In addition, the reference lists of all the included citations were screened. We also searched clinical trial registries for unpublished and in-progress studies.

Table 1. Subject term translations for individual databases

\begin{tabular}{lll}
\hline MEDLINE and Cochrane & PsycINFO & CINAHL \\
\hline Mobile applications & Not available & Mobile applications \\
Computers, handheld & Mobile devices, computer peripheral devices & Computers, handheld \\
Electronic health records & Not available & Electronic health records \\
Cell phone & Cellular phones & Cell phone \\
Surveys and questionnaires & Surveys; questionnaires & Data collection methods \\
Interviews as topic & Interviews & Included in data collection methods \\
Costs and cost analysis & Costs and cost analysis & Costs and cost analysis \\
Data accuracy & Not available & Not available \\
\hline
\end{tabular}

The search strategy reported in the protocol was refined and updated in collaboration with a research librarian. This strategy considered 3 categories: the technology or intervention used (eg, mobile device, mobile phone, mHealth, or EDC), area of application (eg, data collection, demographic and health survey, or large-scale survey), and the outcome of interest (eg, data quality, missing data, and cost-effectiveness). We linked synonyms and controlled vocabulary with Boolean operators
$O R$ and the categories with the operator $A N D$. Textbox 1 presents the search strategy in MEDLINE via Ovid. Appropriate modifications to control for vocabulary and syntax were made to the search strategy for each database (Textbox 1). Additional search strategies for PsycINFO, CINAHL, Web of Science, and Cochrane databases are presented in the supplementary file (Multimedia Appendix 1). All searches were conducted in January 2019. 
Textbox 1. Search strategy in MEDLINE via Ovid.

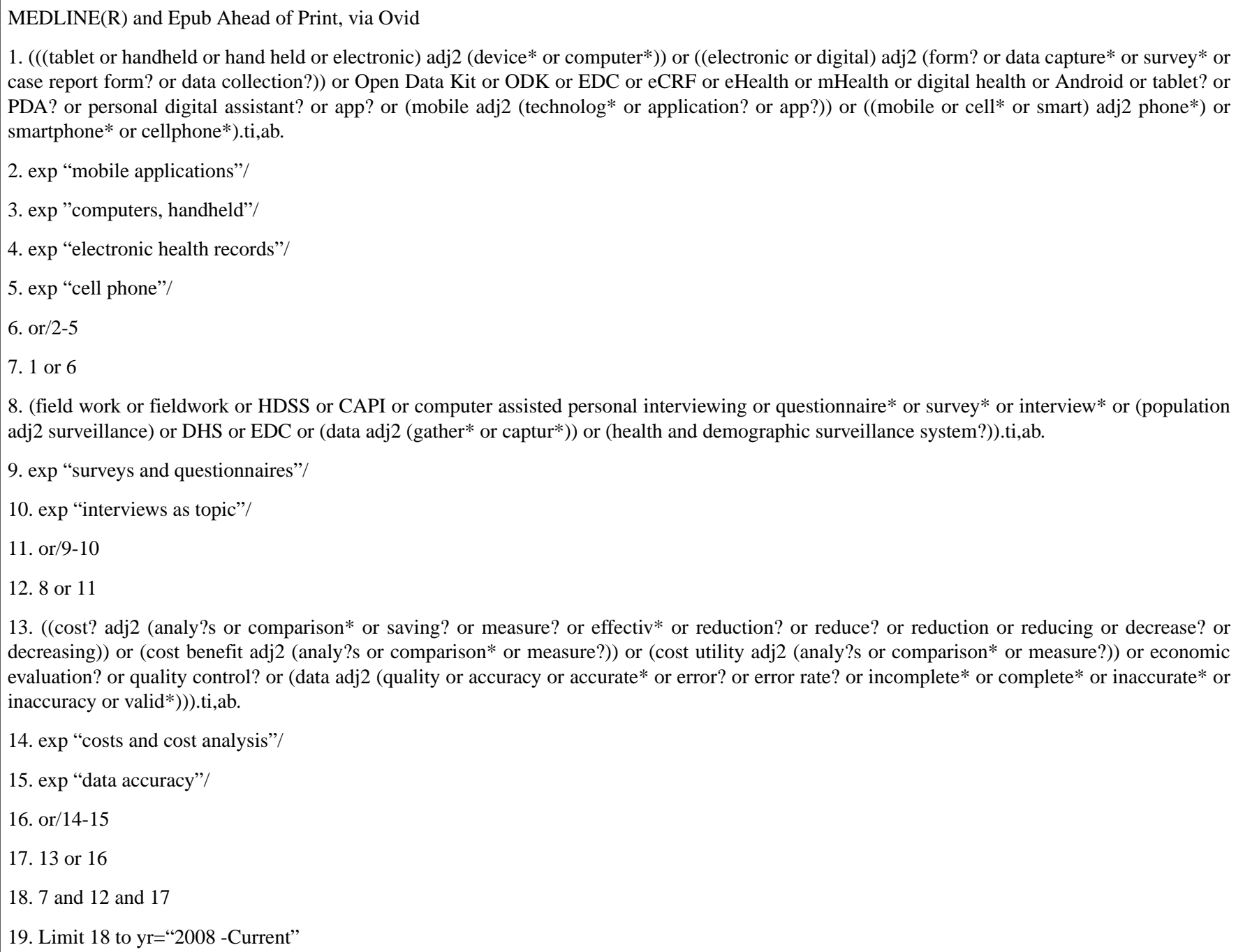

13. ((cost? adj2 (analy?s or comparison* or saving? or measure? or effectiv* or reduction? or reduce? or reduction or reducing or decrease? or decreasing)) or (cost benefit adj2 (analy?s or comparison* or measure?)) or (cost utility adj2 (analy?s or comparison* or measure?)) or economic evaluation? or quality control? or (data adj2 (quality or accuracy or accurate* or error? or error rate? or incomplete* or complete* or inaccurate* or inaccuracy or valid*))).ti,ab.

14. exp "costs and cost analysis"/

15. exp "data accuracy"/

16. or/14-15

17. 13 or 16

18. 7 and 12 and 17

19. Limit 18 to $\mathrm{yr}=$ "2008 -Current"

\section{Study Selection}

We imported all citations from all databases to EndNote for deduplication management and further screening. Although we planned to use the Covidence web-based screening tool to manage the title and abstract screening process, we finally chose the Rayyan QCRI (Qatar Computing Research Institute) screening tool because it is freely available and provides sufficient screening functionalities. Two authors (AZ, MPH in Health Informatics, and TN, MSc in Informatics) independently screened the titles, abstracts, and full text, based on the inclusion criteria. Disagreements and uncertainty on the screening results were first resolved through discussion among the reviewers, followed by consultation with the third (FF, Postdoc in Medical Informatics) and fifth authors (RR, Professor in Medical Informatics). We used a pretested and standardized (through calibration exercise) Microsoft Excel sheet for data extraction based on the inclusion criteria and the objectives of the review.

\section{Data Management and Extraction Process}

Two reviewers extracted the following information from the papers:

- Bibliographic information (authors, titles, journals, and year of publication).
- Characteristics of the intervention (eg, hardware, software, and networking).

- Study methods (design, setting, participants, and sample size).

- Assessed outcomes (data quality, cost-effectiveness, and others).

- Quantitative or qualitative summary of the main findings, including descriptive frequencies and statistical tests.

The full description of the data extraction items can be accessed in the published protocol [33].

\section{Risk of Bias or Quality Assessment}

Randomized controlled trials are suitable for evaluating whether drugs are effective; however, for interventions that involve health care delivery modes, it may not be appropriate or possible to conduct a randomized controlled trial. We aimed to assess the quality of the data in the included studies using parameters such as random sequence generation, allocation concealment, blinding of participants and personnel, blinding of outcome assessment, incomplete outcome data, selective reporting, and other biases. However, the included studies were neither randomized controlled trials nor nonrandomized trials with clinical outcomes; they were mainly prospective comparative experimental studies, cross-sectional studies, or historical 
secondary data record comparisons. The remaining studies were a one-time feasibility study or experience reports from the implementation or use of an EDC tool in public health practice.

\section{Data Synthesis}

There was substantial heterogeneity among the studies concerning the intervention (mobile electronic data capturing tools such as PDA, smartphone, or tablet computer and the app they used), outcome types (error rate and missing or inaccurate data), and level of outcome measurements (sample level, household level, and variable level) of the mHealth interventions and study outcomes.

The studies were found to be noncombinable, and combining these studies would not have been methodologically sound. Consequently, we performed a narrative synthesis of the studies.

\section{Results}

\section{Study Selection and Characteristics}

The search performed in the included databases yielded 3817 results. After deduplication, 2533 results were exported to the
Rayyan QCRI screening tool (Figure 1). Of these, 2500 papers were discarded after title and abstract screening, as these papers clearly did not meet our criteria. Of the 33 full-text papers included, only 14 met the amended inclusion criteria. The original protocol was aimed at including comparative studies that addressed paper-based and electronic tools in the same study, conducted household-level data collection in a community field setting, and reported primary outcomes (data quality or cost-effectiveness) of both data collection tools in the same paper. Only 7 studies (that are heterogeneous) fulfilled these criteria. Due to the limited evidence, we extended our inclusion criteria to cover studies that use the tools in demographic surveys or surveillance systems in a clinical or hospital setting. We also included one-sided study design papers that only reported primary outcomes (cost or data quality) and EDC methods, without formal comparison with paper-based data collection methods. This widening of the scope provided an additional 7 papers ( 3 comparative and 4 noncomparative EDC papers) to bring the total to 14: 10 comparative and 4 noncomparative single-arm studies were included for final full-text extraction (Figure 1). 
Figure 1. Screening process based on the PRISMA (Preferred Reporting Items for Systematic Review and Meta-Analysis) flowchart template.

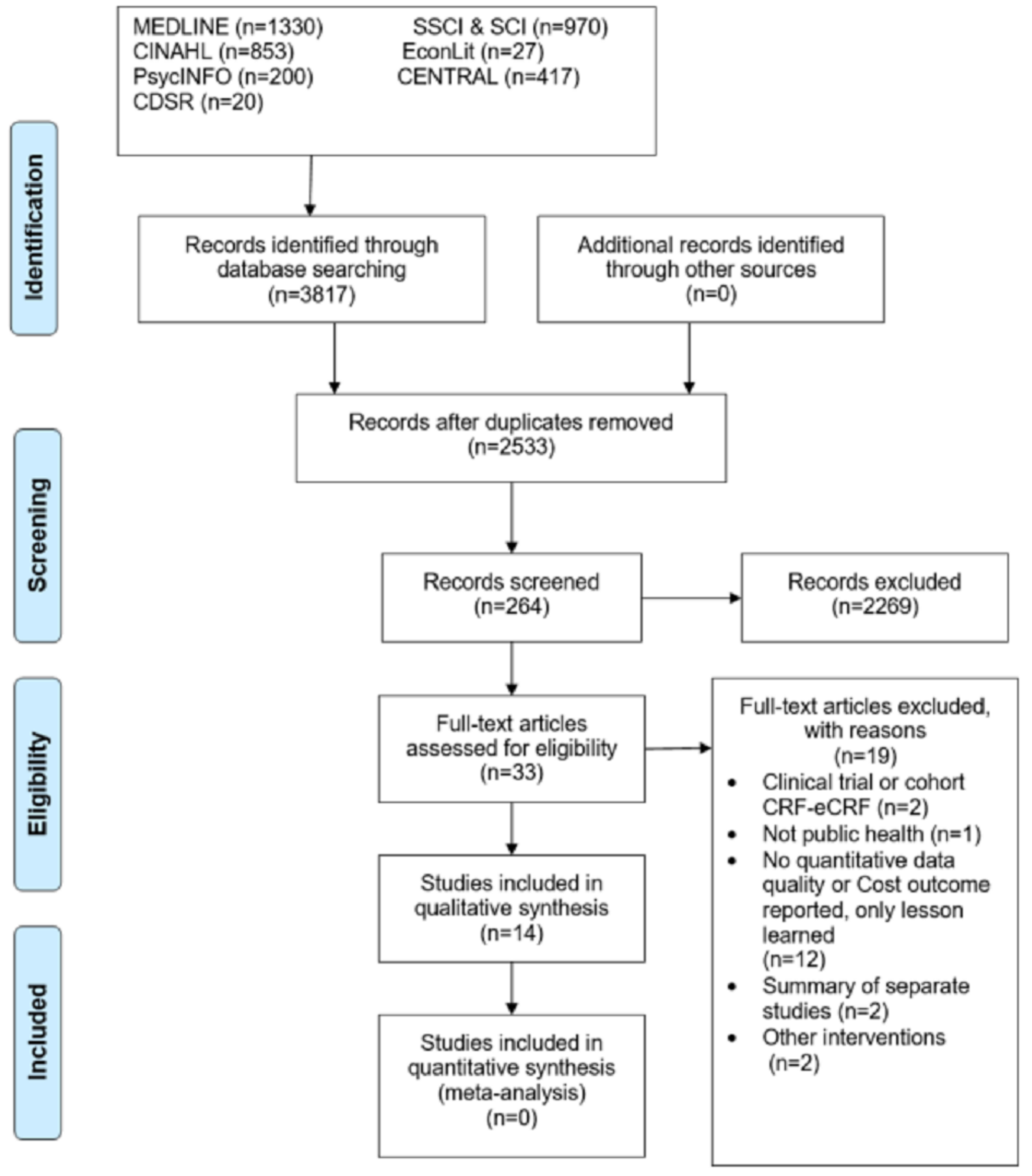

\section{Study Characteristics}

The final systematic synthesis analysis included 14 studies conducted in 12 LMIC. Of these 14, 10 [7,34-42] comparatively assessed the outcomes using paper-based data collection and EDC in the same analysis. Furthermore, 4 papers [43-46] reported either data quality or cost-related outcomes or both in a study conducted using an EDC tool (Table 2). 
Table 2. Study and content characteristics of the included papers

\begin{tabular}{|c|c|c|}
\hline Category & Studies, $\mathrm{n}$ & References \\
\hline \multicolumn{3}{|l|}{ Country $(n=14)$} \\
\hline Kenya & 2 & {$[36,45]$} \\
\hline Ethiopia & 1 & [7] \\
\hline China & 2 & {$[42,43]$} \\
\hline Malawi & 1 & [39] \\
\hline India & 1 & [37] \\
\hline Philippines/Bangladesh & 1 & {$[34]$} \\
\hline Sudan & 1 & {$[35]$} \\
\hline Burkina Faso & 2 & {$[40,44]$} \\
\hline Tanzania & 1 & {$[38]$} \\
\hline South Africa & 1 & {$[41]$} \\
\hline Nigeria & 1 & [46] \\
\hline \multicolumn{3}{|l|}{ Study setting $(\mathrm{n}=10)$} \\
\hline Household community setting & 7 & {$[7,34,35,37-40]$} \\
\hline Clinical/hospital setting & 3 & {$[36,41,42]$} \\
\hline \multicolumn{3}{|l|}{ Comparison of paper-based and electronic data collection $(n=10)$} \\
\hline Both from the same study & 8 & {$[34,35,37-42]$} \\
\hline Compared from 2 studies conducted at different times & 2 & {$[7,36]$} \\
\hline \multicolumn{3}{|l|}{ Purpose of the study design $(n=7)$} \\
\hline Primarily designed to evaluate paper-based and electronic tools & 5 & {$[35,37,39,41,42]$} \\
\hline Secondary byproduct of another primary survey & 2 & {$[7,36]$} \\
\hline \multicolumn{3}{|l|}{ Types of outcomes $(n=10)$} \\
\hline Only data quality outcomes & 1 & [35] \\
\hline Only cost outcomes & 2 & {$[34,40]$} \\
\hline Both cost and data quality outcomes & 7 & {$[7,36-39,41,42]$} \\
\hline \multicolumn{3}{|l|}{ Level of data quality outcome assessment $(n=8)$} \\
\hline Household level & 2 & {$[7,38]$} \\
\hline Questionnaire level & 4 & {$[7,35,41,42]$} \\
\hline Variable level & 3 & {$[36,37,39]$} \\
\hline \multicolumn{3}{|l|}{ Type of data quality outcome comparison $(n=8)$} \\
\hline Missing & 4 & {$[7,36,38,39]$} \\
\hline Inaccurate & 4 & {$[7,36,38,39]$} \\
\hline Mixed (identified as error) & 5 & {$[35,37,38,41,42]$} \\
\hline \multicolumn{3}{|l|}{ Economic evaluation type $(n=11)$} \\
\hline Complete input cost & 3 & {$[36,38,40]$} \\
\hline Partial (differential) cost & 8 & {$[7,37,39,41-43,45,46]$} \\
\hline \multicolumn{3}{|l|}{ Usability/user preference evaluation $(n=14)$} \\
\hline Reported after formal evaluation & 6 & {$[7,34,37-39,42]$} \\
\hline Reported with informal discussion & 1 & [36] \\
\hline No user evaluation information & 7 & {$[35,40,41,43-46]$} \\
\hline \multicolumn{3}{|l|}{ Study, intervention, or evaluation year $(n=10)$} \\
\hline $2008-2012$ & 5 & {$[36,38,41,42,44]$} \\
\hline
\end{tabular}




\begin{tabular}{lll}
\hline Category & Studies, $\mathrm{n}$ & References \\
\hline 2013-2018 & 5 & {$[34,35,37,43,46]$} \\
Publication year (n=14) & & \\
2008-2012 & 2 & {$[42,46]$} \\
$2013-2018$ & 12 & {$[7,34-41,43,45,46]$} \\
\hline
\end{tabular}

Furthermore, 5 studies $[35,37,39,41,42]$ were primarily intended to evaluate and compare data quality and cost-related outcomes from a prospective study design using paper-based and electronic tools. The remaining 5 papers $[7,34,36,38,40]$ reported the outcomes from previous technology utilization experiences. The reported outcomes were not primarily intended to evaluate the tools; rather, data quality and cost-related outcomes were extracted from surveys at different times.

Regarding the settings, 7 studies [7,34,35,37-40] in the comparative group included data from a household-level survey, and 3 studies $[36,41,42]$ conducted surveys in clinical settings or research centers.

\section{Types of Outcomes}

\section{Data Quality Outcomes}

Data quality outcomes, as defined in the methods section, comprise the frequency of errors (incomplete, missing, or inaccurate items) on 3 levels, based on the reported outcomes at the household, questionnaire, and variable levels. At the household level, the incidence of 1 or more errors among the total number of households included in the surveys and data analysis were reported in 2 papers [7,38]. Similarly, the frequency of 1 or more errors per complete questionnaire, regarded as a questionnaire-level error, was reported in 4 studies $[7,35,41,42]$. At a variable level, a count of the errors in a complete set of questions or variables in questionnaires measured as a variable error were mentioned in 3 papers (Figure 2) $[36,37,39]$.

Figure 2. Forest plot comparison of heterogeneity characteristics of the data quality outcomes.

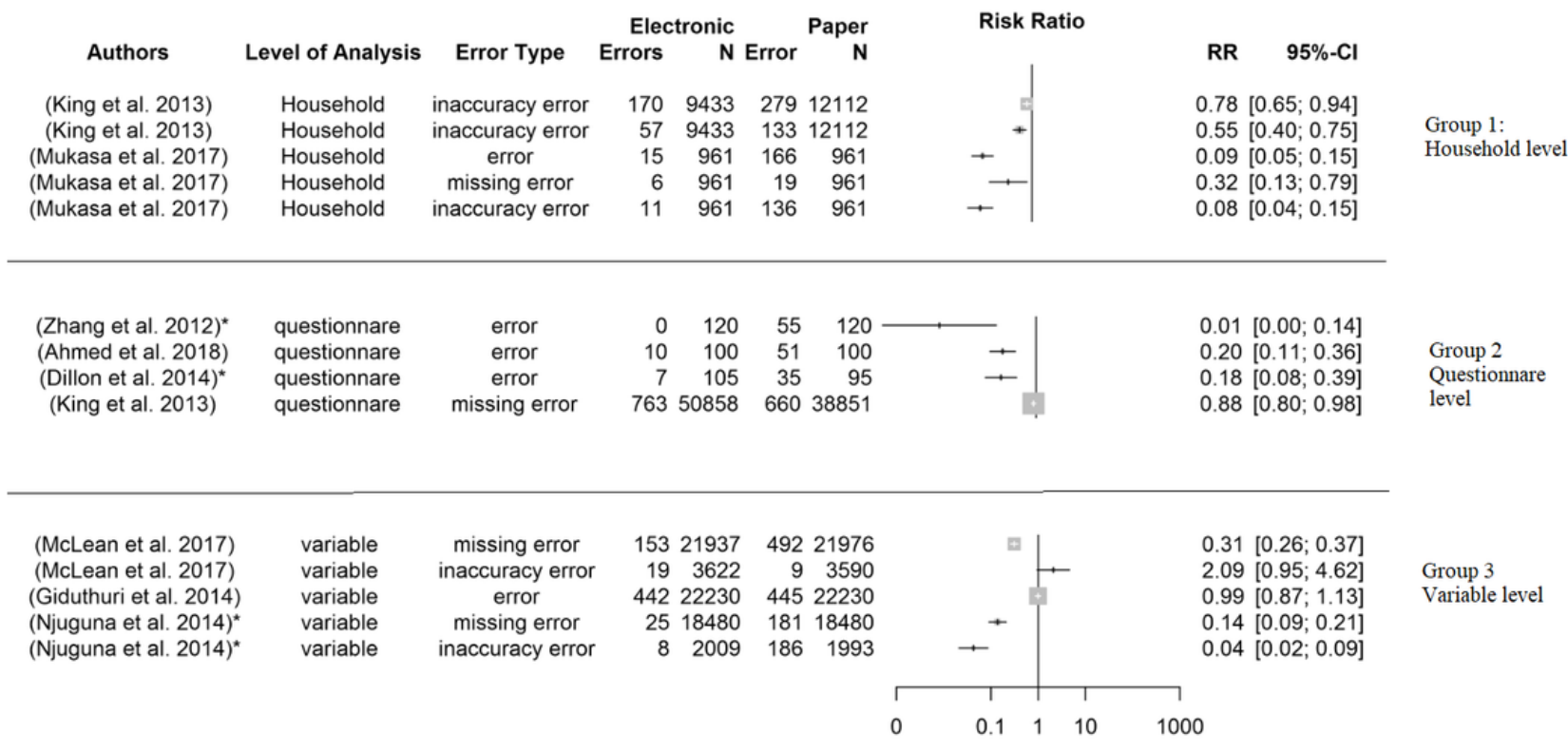

The cost of implementing electronic and paper-based data collection processes was estimated by most studies (12 out of 14). The majority of these estimated the partial or differential costs unique to that study and its EDC [7,37,39,41-43,45,46]. Only 3 papers listed the full implementation cost $[36,38,40]$ for both the study and the EDC. Except 2 studies that compared costs per correctly entered data observation or error-free databases [39,41], none attempted to link the data quality outcome measures to the cost inputs.

\section{Effect on Data Quality: Missing Data and Inaccuracy Errors}

Errors are reported in terms of missing and inaccurate data in 4 studies [7,36,38,39], while a combination of both errors as a single error indicator is reported in 4 others $[35,37,38,42]$ (Table $3)$. 
Table 3. Extracted data quality outcomes.

\begin{tabular}{|c|c|c|c|c|c|c|c|}
\hline $\begin{array}{l}\text { Study and } \\
\text { country }\end{array}$ & $\begin{array}{l}\text { Type of compari- } \\
\text { son, study design, } \\
\text { and setting }^{\text {a }}\end{array}$ & $\begin{array}{l}\text { Study population } \\
\text { and sample unit }\end{array}$ & $\begin{array}{l}\text { Interven- } \\
\text { tion: de- } \\
\text { vice or app }\end{array}$ & $\begin{array}{l}\text { Methods of paper- } \\
\text { based and electron- } \\
\text { ic tool administra- } \\
\text { tion }\end{array}$ & $\begin{array}{l}\text { Outcome measurement } \\
\text { characteristics }^{\mathrm{b}}\end{array}$ & Result & $\begin{array}{l}\text { Biases (selec- } \\
\text { tion, informa- } \\
\text { tion, or con- } \\
\text { founding) }\end{array}$ \\
\hline $\begin{array}{l}\text { Ahmed et } \\
\text { al [35], Su- } \\
\text { dan }\end{array}$ & $\begin{array}{l}\text { B: Exploratory pi- } \\
\text { lot study nested } \\
\text { and experimented } \\
\text { in cross-sectional } \\
\text { household surveys }\end{array}$ & $\begin{array}{l}\text { - } \\
\text { - } \text { responde unit: } \\
\text { - } \\
\text { Sample size: } \\
100 \text { for each } \\
\left(\mathrm{PPDC}^{\mathrm{c}} \text { and }\right. \\
\left.\mathrm{EDC}^{\mathrm{d}}\right)\end{array}$ & $\begin{array}{l}\text { EDC: } \\
\text { Smart- } \\
\text { phone } \\
\text { ODK }^{\mathrm{e}} \text { app }\end{array}$ & $\begin{array}{l}\text { Daily sample ran- } \\
\text { domization for } \\
\text { EDC or PPDC. } 1 \\
\text { respondent simulta- } \\
\text { neously inter- } \\
\text { viewed by } 2 \text { inter- } \\
\text { viewers with differ- } \\
\text { ent tools (PPDC } \\
\text { and EDC) }\end{array}$ & $\begin{array}{l}\text { A: Data quality er- } \\
\text { rors, questions } \\
\text { with no answers, } \\
\text { or wrong use of } \\
\text { the skip pattern } \\
\text { B: After data entry } \\
\text { C: Errors mea- } \\
\text { sured at the ques- } \\
\text { tionnaire level } \\
\text { D: Used similar } \\
\text { questionnaires } \\
\text { (BOLD }{ }^{\text {f }} \text { survey } \\
\text { core question- } \\
\text { naire) }\end{array}$ & $\begin{array}{ll}\text { - } & \text { PPDC: } 51 \text { er- } \\
& \text { rors from } 100 \\
\text { question- } \\
\text { naires } \\
\text { - } & \text { EDC: } 10 \text { er- } \\
\text { rors from } 100 \\
\text { electronic } \\
\text { forms } \\
\text { 83\% errors in } \\
\text { PPDC }\end{array}$ & $\begin{array}{l}\text { Selection } \\
\text { and informa- } \\
\text { tion }\end{array}$ \\
\hline $\begin{array}{l}\text { King et al } \\
\text { [7], } \\
\text { Ethiopia }\end{array}$ & $\begin{array}{l}\text { A: Pre- and postde- } \\
\text { sign in full EDC } \\
\text { implementation } \\
\text { evaluation in cross- } \\
\text { sectional house- } \\
\text { hold surveys }\end{array}$ & 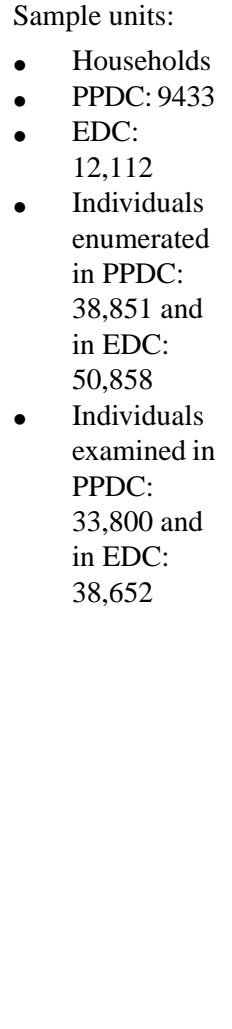 & $\begin{array}{l}\text { EDC: For } \\
\text { example, } \\
\text { tablet com- } \\
\text { puter or } \\
\text { self-devel- } \\
\text { oped An- } \\
\text { droid app }\end{array}$ & $\begin{array}{l}\text { Paper and electron- } \\
\text { ic surveys at differ- } \\
\text { ent places and } \\
\text { times, } 1 \text { data collec- } \\
\text { tor at a time }\end{array}$ & $\begin{array}{l}\text { A: Data quality, } \\
\text { percentage of indi- } \\
\text { viduals enumerat- } \\
\text { ed with at least } 1 \\
\text { blank field in the } \\
\text { census record } \\
\text { Percentage of } \\
\text { households with } \\
\text { incorrect unique } \\
\text { identifying num- } \\
\text { ber (inaccuracy at } \\
\text { the household lev- } \\
\text { el) } \\
\text { GPS with blank } \\
\text { entries (missing at } \\
\text { the household lev- } \\
\text { el) } \\
\text { The proportion of } \\
\text { total time } \\
\text { B: After data entry } \\
\text { (raw data sets) } \\
\text { C: At the question- } \\
\text { naire and house- } \\
\text { hold levels } \\
\text { D: Used the same } \\
\text { questionnaire } \\
\text { (Trachoma Impact } \\
\text { Evaluation Survey } \\
\text { questionnaire) }\end{array}$ & $\begin{array}{l}\text { PPDC: Miss- } \\
\text { ing rate at the } \\
\text { questionnaire } \\
\text { level is } 1.7 \% \\
\text { EDC: Miss- } \\
\text { ing } 1.5 \% \text { at } \\
\text { P=.01 } \\
\text { PPDC: Miss- } \\
\text { ing rate in } \\
\text { GPS data } \\
\text { 0.6\% } \\
\text { (N=9263) } \\
\text { EDC: Miss- } \\
\text { ing rate in } \\
\text { GPS } 1.1 \% \\
\text { (N=12,064; } \\
\text { P<.01; per- } \\
\text { son-days) } \\
\text { PPDC: Pro- } \\
\text { portion of to- } \\
\text { tal time } 790 \\
\text { person-days } \\
\text { EDC: Propor- } \\
\text { tion of total } \\
\text { time 511 per- } \\
\text { son-days }\end{array}$ & $\begin{array}{l}\text { Selection, in- } \\
\text { formation, } \\
\text { and con- } \\
\text { founding }\end{array}$ \\
\hline
\end{tabular}




\begin{tabular}{|c|c|c|c|c|c|c|c|}
\hline $\begin{array}{l}\text { Study and } \\
\text { country }\end{array}$ & $\begin{array}{l}\text { Type of compari- } \\
\text { son, study design, } \\
\text { and setting }^{\mathrm{a}}\end{array}$ & $\begin{array}{l}\text { Study population } \\
\text { and sample unit }\end{array}$ & $\begin{array}{l}\text { Interven- } \\
\text { tion: de- } \\
\text { vice or app }\end{array}$ & $\begin{array}{l}\text { Methods of paper- } \\
\text { based and electron- } \\
\text { ic tool administra- } \\
\text { tion }\end{array}$ & $\begin{array}{l}\text { Outcome measurement } \\
\text { characteristics }^{b}\end{array}$ & Result & $\begin{array}{l}\text { Biases (selec- } \\
\text { tion, informa- } \\
\text { tion, or con- } \\
\text { founding) }\end{array}$ \\
\hline $\begin{array}{l}\text { McLean et } \\
\text { al [39], } \\
\text { Malawi }\end{array}$ & $\begin{array}{l}\text { A: Prospective } \\
\text { evaluation nested } \\
\text { and experimented } \\
\text { in cross-sectional } \\
\text { household surveys } \\
\text { at } \text { HDSS }^{\mathrm{g}} \text { sites }\end{array}$ & $\begin{array}{ll}\text { - } & \text { PPDC: } 426 \\
& \text { respondents } \\
\text { - } & \text { EDC: } 558 \\
& \text { respondents } \\
\text { - } & \text { Time: } 3 \\
\text { weeks }\end{array}$ & $\begin{array}{l}\text { EDC: } \\
\text { Tablet } \\
\text { computer } \\
\text { and smart- } \\
\text { phone, } \\
\text { ODK app }\end{array}$ & $\begin{array}{l}\text { Independent paral- } \\
\text { lel EDC or PPDC, } \\
1 \text { data collector at } \\
\text { a time }\end{array}$ & $\begin{array}{l}\text { A: Missing data } \\
\text { defined as not } \\
\text { asked (blank; dis- } \\
\text { counting not appli- } \\
\text { cable blank ques- } \\
\text { tions) or as blank } \\
\text { and entered as un- } \\
\text { known combined, } \\
\text { Internal validity: } \\
\text { defined as a field } \\
\text { with an impossible } \\
\text { or inconsistent } \\
\text { value and time for } \\
\text { submission } \\
\text { B: Not clear } \\
\text { C: Variable-level } \\
\text { error count } \\
\text { D: Used different } \\
\text { questionnaires }\end{array}$ & 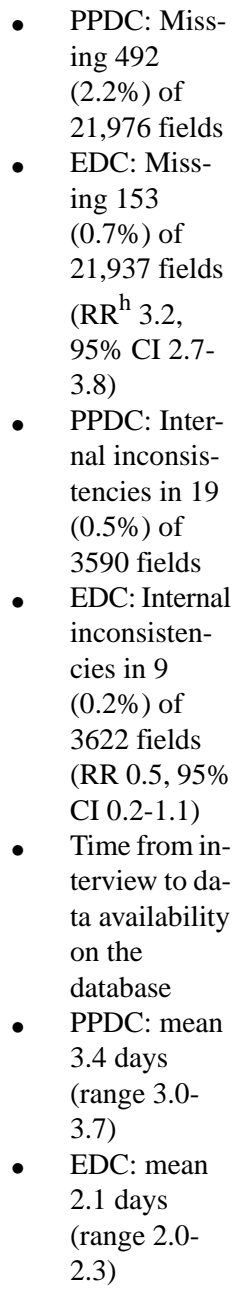 & $\begin{array}{l}\text { Selection } \\
\text { and informa- } \\
\text { tion }\end{array}$ \\
\hline $\begin{array}{l}\text { Giduthuri } \\
\text { et al [37], } \\
\text { India }\end{array}$ & $\begin{array}{l}\text { A: Prospective ex- } \\
\text { perimental compar- } \\
\text { ative study de- } \\
\text { signed for house- } \\
\text { holds selected ran- } \\
\text { domly }\end{array}$ & $\begin{array}{l}\text { - } 98 \text { inter- } \\
\text { views in } \\
\text { EDC and } \\
\text { PPDC }\end{array}$ & $\begin{array}{l}\text { EDC: } \\
\text { Tablet } \\
\text { comput- } \\
\text { er/ODK } \\
\text { app }\end{array}$ & $\begin{array}{l}\text { A respondent simul- } \\
\text { taneously inter- } \\
\text { viewed by } 2 \text { inter- } \\
\text { viewers with differ- } \\
\text { ent tools (PPDC } \\
\text { and EDC) }\end{array}$ & $\begin{array}{l}\text { A: Discrepancies } \\
\text { with reference to } \\
\text { device-attributable } \\
\text { errors } \\
\text { Paper entries incor- } \\
\text { rect or missing } \\
\text { and tablet entries } \\
\text { missing because } \\
\text { paper interviewer } \\
\text { (lead) did not fol- } \\
\text { low the skip logic } \\
\text { Tablet entries in- } \\
\text { correct or missing, } \\
\text { paper entries miss- } \\
\text { ing because tablet } \\
\text { interviewer's } \\
\text { (lead) skip logic } \\
\text { was considered an } \\
\text { error of EDC } \\
\text { B: During data en- } \\
\text { try } \\
\text { C: Variable-level } \\
\text { error count } \\
\text { D: Used the same } \\
\text { questionnaire }\end{array}$ & $\begin{array}{ll}\text { - } & \text { PPDC: Mean } \\
\text { number of pa- } \\
\text { per-at- } \\
\text { tributable er- } \\
\text { rors } 4.68 \\
(445 / 22,230, \\
2.01 \%) \\
\text { EDC: Mean } \\
\text { number of } \\
\text { tablet-at- } \\
\text { tributable er- } \\
\text { rors } 4.65 \\
(442 / 22,230, \\
1.99 \%)\end{array}$ & $\begin{array}{l}\text { Selection, in- } \\
\text { formation, } \\
\text { and con- } \\
\text { founding }\end{array}$ \\
\hline
\end{tabular}




\begin{tabular}{|c|c|c|c|c|c|c|c|}
\hline $\begin{array}{l}\text { Study and } \\
\text { country }\end{array}$ & $\begin{array}{l}\text { Type of compari- } \\
\text { son, study design, } \\
\text { and setting }^{\mathrm{a}}\end{array}$ & $\begin{array}{l}\text { Study population } \\
\text { and sample unit }\end{array}$ & $\begin{array}{l}\text { Interven- } \\
\text { tion: de- } \\
\text { vice or app }\end{array}$ & $\begin{array}{l}\text { Methods of paper- } \\
\text { based and electron- } \\
\text { ic tool administra- } \\
\text { tion }\end{array}$ & $\begin{array}{l}\text { Outcome measurement } \\
\text { characteristics }^{b}\end{array}$ & Result & $\begin{array}{l}\text { Biases (selec- } \\
\text { tion, informa- } \\
\text { tion, or con- } \\
\text { founding) }\end{array}$ \\
\hline $\begin{array}{l}\text { Mukasa et } \\
\text { al [38], } \\
\text { Tanzania }\end{array}$ & $\begin{array}{l}\text { A: Retrospective } \\
\text { record review of } \\
\text { household survey } \\
\text { data }\end{array}$ & $\begin{array}{l}961 \text { house- } \\
\text { holds for } \\
\text { EDC and } \\
\text { PPDC }\end{array}$ & $\begin{array}{l}\text { EDC or } \\
\text { PDA } \\
\text { BlackBerry } \\
\text { customized } \\
\text { HRS }^{\mathrm{i}}\end{array}$ & $\begin{array}{l}\text { Repeated survey, } \\
\text { PPDC first, fol- } \\
\text { lowed by EDC, } 1 \\
\text { data collector at a } \\
\text { time }\end{array}$ & $\begin{array}{l}\text { A: Error category } \\
\text { from the database: } \\
\text { accuracy, logic, } \\
\text { and consistency; } \\
\text { range; and com- } \\
\text { pleteness and } \\
\text { missing values } \\
\text { B: After data entry } \\
\text { from database } \\
\text { C: Error identified } \\
\text { at the household } \\
\text { level } \\
\text { D: Used the same } \\
\text { questionnaire }\end{array}$ & $\begin{array}{ll}\text { - } & \text { Households } \\
\text { with errors } \\
\text { - } & \text { PPDC: } 166 \\
& (17 \%) \\
\text { - } & \text { EDC: } 15 \\
& (2 \%) \\
\text { - } & \text { PPDC more } \\
\text { likely with ac- } \\
\text { curacy errors } \\
(79 \% ; 95 \% \\
\text { CI } 72 \%- \\
86 \%) ; \text { EDC: } \\
(58 \% ; 95 \% \\
\text { CI } 29 \%-87 \%) \\
\text { Errors in } \\
\text { EDC more } \\
\text { likely to be } \\
\text { related to } \\
\text { completeness } \\
\text { (32\%; } 95 \% \\
\text { CI } 12 \%-56 \%) \\
\text { than in PPDC } \\
\text { (11\%; } 95 \% \\
\text { CI } 7 \%-17 \%)\end{array}$ & $\begin{array}{l}\text { Selection, in- } \\
\text { formation, } \\
\text { and con- } \\
\text { founding }\end{array}$ \\
\hline $\begin{array}{l}\text { Zhang et al } \\
\text { [42], China }\end{array}$ & $\begin{array}{l}\text { B: Prospective } \\
\text { comparison study } \\
\text { conducted in a } \\
\text { clinic }\end{array}$ & $\begin{array}{l}60 \text { mothers } \\
\text { for each tool } \\
\text { (EDC and } \\
\text { PPDC) }\end{array}$ & $\begin{array}{l}\text { EDC: } \\
\text { smart- } \\
\text { phones, } \\
\text { leased soft- } \\
\text { ware }\end{array}$ & $\begin{array}{l}\text { A respondent simul- } \\
\text { taneously inter- } \\
\text { viewed by } 2 \text { inter- } \\
\text { viewers with same } \\
\text { tools (PPDC or } \\
\text { EDC). Random } \\
\text { assignation of re- } \\
\text { spondents to one of } \\
\text { the tools }\end{array}$ & $\begin{array}{l}\text { A: Data quality er- } \\
\text { rors; missing con- } \\
\text { firmation of de- } \\
\text { fault option, sur- } \\
\text { vey date, missed } \\
\text { questions, } 2 \text { op- } \\
\text { tions circled, } \\
\text { wrong options } \\
\text { chosen, date, ID; } \\
\text { database comple- } \\
\text { tion (data entry, } \\
\text { checking, and data } \\
\text { cleaning) } \\
\text { B: Before and af- } \\
\text { ter data entry } \\
\text { C: Questionnaire- } \\
\text { level error count } \\
\text { D: Used the same } \\
\text { questionnaire }\end{array}$ & 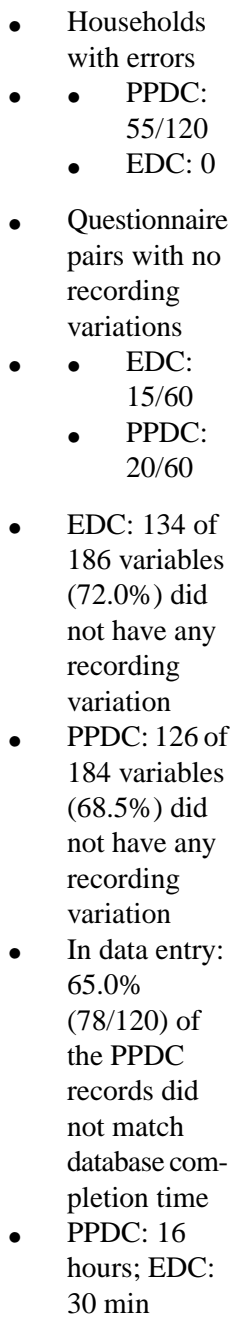 & $\begin{array}{l}\text { Selection, in- } \\
\text { formation, } \\
\text { and con- } \\
\text { founding }\end{array}$ \\
\hline
\end{tabular}




\begin{tabular}{|c|c|c|c|c|c|c|c|}
\hline $\begin{array}{l}\text { Study and } \\
\text { country }\end{array}$ & $\begin{array}{l}\text { Type of compari- } \\
\text { son, study design, } \\
\text { and setting }^{\text {a }}\end{array}$ & $\begin{array}{l}\text { Study population } \\
\text { and sample unit }\end{array}$ & $\begin{array}{l}\text { Interven- } \\
\text { tion: de- } \\
\text { vice or app }\end{array}$ & $\begin{array}{l}\text { Methods of paper- } \\
\text { based and electron- } \\
\text { ic tool administra- } \\
\text { tion }\end{array}$ & $\begin{array}{l}\text { Outcome measurement } \\
\text { characteristics }^{b}\end{array}$ & Result & $\begin{array}{l}\text { Biases (selec- } \\
\text { tion, informa- } \\
\text { tion, or con- } \\
\text { founding) }\end{array}$ \\
\hline $\begin{array}{l}\text { Njuguna et } \\
\text { al [36], } \\
\text { Kenya }\end{array}$ & $\begin{array}{l}\text { A: Pre- and } \\
\text { postimplementa- } \\
\text { tion evaluation in } \\
\text { hospital-based } \\
\text { surveillance data } \\
\text { collection }\end{array}$ & $\begin{array}{l}\text { EDC: } 880 \\
\text { question- } \\
\text { naires (May } \\
\text { 2011-June } \\
\text { 2012) } \\
\text { PPDC: } 880 \\
\text { question- } \\
\text { naires (Jan- } \\
\text { uary 2010- } \\
\text { June 2011) }\end{array}$ & $\begin{array}{l}\text { EDC: } \\
\text { smart- } \\
\text { phones, } \\
\text { FAST }^{\mathrm{j}} \text { app }\end{array}$ & $\begin{array}{l}\text { Paper and electron- } \\
\text { ic surveys at the } \\
\text { same place but dif- } \\
\text { ferent times, } 1 \text { data } \\
\text { collector at a time }\end{array}$ & $\begin{array}{l}\text { A: Incomplete } \\
\text { records or vari- } \\
\text { ables in selected } \\
\text { questions in a } \\
\text { questionnaire; } \\
\text { questions requir- } \\
\text { ing responses with } \\
\text { or without pro- } \\
\text { grammed checks } \\
\text { on the smartphone } \\
\text { version; percent- } \\
\text { age of erroneous } \\
\text { and inconsistent } \\
\text { responses in the } \\
\text { questionnaires } \\
\text { B: Error count } \\
\text { from original re- } \\
\text { sponses in the pa- } \\
\text { per } \\
\text { C: Variable-level } \\
\text { error count } \\
\text { D: Used the same } \\
\text { questionnaire }\end{array}$ & $\begin{array}{ll}\text { - } & \text { Missing error: } \\
\text { - } & \text { EDC: } \\
& \text { 25/18,480 } \\
& \text { PPDC: } \\
& \text { 181/18,299 } \\
& \\
\text { - } & \text { Inaccuracy er- } \\
\text { - ror: } & \\
\text { - } & \text { EDC: } \\
& 8 / 2009 \\
& \text { PPDC: } \\
& 186 / 1807\end{array}$ & $\begin{array}{l}\text { Selection, in- } \\
\text { formation, } \\
\text { and con- } \\
\text { founding }\end{array}$ \\
\hline $\begin{array}{l}\text { Dillon et al } \\
\text { [14], South } \\
\text { Africa }\end{array}$ & $\begin{array}{l}\text { B: Prospective data } \\
\text { collection in case } \\
\text { control study in a } \\
\text { hospital setting }\end{array}$ & $\begin{array}{ll}\text { - } & \text { EDC: } 105 \\
& \text { respondents } \\
\text { - } & \text { PPDC: } 95 \\
& \text { respondents }\end{array}$ & $\begin{array}{l}\text { EDC: } \\
\text { tablet com- } \\
\text { puter, self- } \\
\text { developed } \\
\text { app }\end{array}$ & $\begin{array}{l}\text { One data collector } \\
\text { interviewed all re- } \\
\text { spondents either } \\
\text { with paper or with } \\
\text { electronic tools in } \\
\text { a random order }\end{array}$ & $\begin{array}{l}\text { A: Data collection } \\
\text { errors defined as } \\
\text { nonsensical or im- } \\
\text { possible inputs, } \\
\text { missing data, or } \\
\text { inputs inconsistent } \\
\text { or incompatible } \\
\text { with previous re- } \\
\text { sponses during the } \\
\text { interview; minor } \\
\text { errors classified as } \\
\text { differences of } 1 \\
\text { year or less in date } \\
\text { calculations. Ma- } \\
\text { jor errors classi- } \\
\text { fied as all other er- } \\
\text { ror types } \\
\text { B: Not clear } \\
\text { C: Variable-level } \\
\text { error count } \\
\text { D: Used similar } \\
\text { questionnaires }\end{array}$ & $\begin{array}{l}\text { - } \begin{array}{l}\text { Overall num- } \\
\text { ber of errors }\end{array} \\
\text { per } 100 \text { ques- } \\
\text { tions } \\
\text { - } \quad \text { EDC: } 0.17 \text { er- } \\
\text { rors; PPDC: } \\
0.73 \text { errors } \\
\text { P<.001 } \\
\text { Interview du- } \\
\text { ration (EDC: } \\
\text { 5.4 min; } \\
\text { PPDC: } 5.6 \\
\text { min) }\end{array}$ & $\begin{array}{l}\text { Selection, in- } \\
\text { formation, } \\
\text { and con- } \\
\text { founding }\end{array}$ \\
\hline
\end{tabular}

\footnotetext{
${ }^{\mathrm{a}} \mathrm{A}$ : full implementation; B: pilot testing.

${ }^{\mathrm{b}} \mathrm{A}$ : type of outcome; B: stage of error assessment for paper questionnaire; C: error measurement level; D: questionnaire name/s similarities.

${ }^{\mathrm{c}} \mathrm{PPDC}$ : pen and paper data collection.

${ }^{\mathrm{d}} \mathrm{EDC}$ : electronic data collection.

eODK: Open Data Kit.

${ }^{f}$ BOLD: Burden of Obstructive Lung Disease.

${ }^{\mathrm{g}} \mathrm{HDSS}$ : health and demographic surveillance systems.

${ }^{\mathrm{h}} \mathrm{RR}$ : risk ratio.

${ }^{\mathrm{i}}$ HRS: Household Registration System.

${ }^{\mathrm{j}}$ FAST: Field Adapted Survey Toolkit.
}

An exploratory pilot study nested and experimented in a cross-sectional household survey in Sudan compared data quality errors, questions with no answers, or incorrect use of the skip pattern in 100 convenience samples. A pair of data collectors simultaneously interviewed each respondent -1 with pen and paper and 1 with electronic tools-and recorded the data 
separately. In the paper-based data collection, 51 of the 100 questions had one or more errors, compared with 10 errors in the electronically submitted forms [35].

A study in India by Giduthuri et al [37] also compared error rates between paper-based and electronically collected data from a comparative prospective experimental study. The data collectors were randomly assigned to use either pen and paper or EDC tools while interviewing each respondent simultaneously. Audio-recorded data during the survey were used as a reference to compare discrepancies and device-attributable errors. According to the reference, paper errors indicate when a paper entry was incorrect and when a tablet entry was missing because the paper-based tool interviewer (lead) did not follow the skip logic. Furthermore, tablet entries were incorrect or missing and paper entries were missing because of the electronic tool interviewer's (lead) skip logic was considered an error of the electronic data collection tool. The mean number of paper-attributable errors was 4.68 $(445 / 22,230,2.01 \%)$, while the mean number of tablet-attributable errors was 4.65 (442/22,230, 1.99\%); thus, no significant differences were observed [37].

A study in China compared smartphone and paper-based data collection in an infant feeding practice survey conducted in rural clinics. Purposive sampling techniques were used to select 120 mothers, 60 per survey tool group. Two data collectors with the same tool (paper or electronic) interviewed 60 mothers in random order, yielding 120 records for each tool. For the paper-based questionnaire, 55 of 120 questionnaires had 1 or more errors or missing data. The most frequent error was a missing confirmation of the default option, which was observed 156 times in 49 questionnaires. No missing error was reported for the EDC tool group [42]. The mean duration of an interview was 10.22 (SD 2.17) min for the smartphone method and 10.83 (SD 2.94) min for the pen and paper method. Moreover, database completion took 16 hours (including data entry, checking, and data cleaning) for pen and paper data collection (PPDC), while it took half an hour for EDC [42].

A prospective evaluation experiment conducted a nested, ongoing, cross-sectional household survey at HDSS sites in Malawi. In 3 weeks, 426 interviews with PPDC and 558 interviews with EDC were conducted. Data collectors independently interviewed different households in a 1 data collector in 1 home mode. Missing data were defined as not asked (blank; discounting not applicable blank questions) or as blank and entered as unknown combined. Internal validity was defined as a field with an impossible or inconsistent value and time for submission. In paper questionnaires, missing data were reported in $492(2.2 \%)$ of 21,976 fields, compared with 153 $(0.7 \%)$ of 21,937 fields in electronic forms (risk ratio [RR] 3.2, 95\% CI 2.7-3.8). Internal inconsistencies were found in 19
(0.5\%) of 3590 fields collected by PPDC compared to $9(0.2 \%)$ of 3622 fields for EDC (RR 0.5, 95\% CI 0.2-1.1) [39]. Moreover, the mean data availability duration in databases was 3.4 days (95\% CI 3.0-3.7) in PPDC compared with 2.1 days (95\% CI 2.0-2.3) for EDC. The mean number of interviews per day was similar for both groups at 10.7 (95\% CI 8.7-12.6) for PPDC and 11.8 (95\% CI 8.1-15.5) for EDC [39].

A tablet computer-based data collection system was implemented in a large-scale study of trachoma impact assessment surveys in Ethiopia [7]. Data quality outcomes were compared with a similar paper-based survey conducted 7 months earlier in a different part of the country. The sampling units were households (PPDC: 9433 vs EDC: 12,112), and the study enumerated 38,851 individuals in the PPDC survey and 50,858 in the EDC survey. Individuals enumerated with at least 1 blank field in a single respondent response were defined as missing data (1.7\% for PPDC vs $1.5 \%$ for EDC; $\mathrm{P}=.01)$. Missing data at the household level for GPS with blank entries was also reported (EDC: $1.1 \%$ vs PPDC: $0.6 \%$; $\mathrm{P}<.01$ ). Inaccuracy errors were defined only in a percentage of households with an incorrect unique identifying number (PPDC: $2.3 \%$ vs EDC: $1.8 \%$ ). Data entry and analysis were done in less than 1 day for EDC, while it took 14 days for data entry and an additional 5 days for double data entry discrepancy checks for PPDC [7].

Apart from the above studies, interview time or mean data availability duration in the databases were also reported in some papers. A study in Kenya reported faster data upload to a central database in EDC and a median duration for data upload of 7 days (range 1-13 days) after data collection for EDC and 21 days (range 4-56 days) for PPDC $(\mathrm{P}<.01)$ [36]. A combined report from Indonesia and the Philippines showed that the median time between data collection and data entry for PPDC surveys was approximately 3 months, compared with 2 days for EDC [34]. Time analysis from a large-scale survey in Tanzania reported that the median duration of an enumeration session per household was $9.4 \mathrm{~min}$ (90\% central range 6.4-12.2) for paper surveys and $8.3 \mathrm{~min}(6.1-12.0)$ for electronic surveys $(\mathrm{P}=.01)[38]$.

\section{Effect on Cost-effectiveness}

Most of the studies reported cost analysis for the expenses incurred to conduct surveys using paper-based and electronic tools. However, the included studies varied significantly in the types and level of cost analysis reported in their groups. Most of the recommendations from the Consolidated Health Economic Evaluation Reporting Standards (CHEERS) statement [47] were not included. In Table 4, we provide basic information on the study and country, analytic method and model, participants per intervention, time horizon, discount rate, currency, included cost inputs, cost ranges, outcomes, consequence, and conclusion information. 
Table 4. Extracted cost information (based on Consolidated Health Economic Evaluation Reporting Standards evaluation template)

\begin{tabular}{|c|c|c|c|c|c|c|c|}
\hline $\begin{array}{l}\text { Study and } \\
\text { country }\end{array}$ & $\begin{array}{l}\text { Analytic } \\
\text { method or } \\
\text { model }\end{array}$ & $\begin{array}{l}\text { Interventions } \\
\text { studied or popula- } \\
\text { tion per group } \\
\left(1=\mathrm{PPDC}^{\mathrm{a}} \text {; }\right. \\
\left.2=\mathrm{EDC}^{\mathrm{b}}\right)\end{array}$ & $\begin{array}{l}\text { Time horizon, } \\
\text { discount rate, } \\
\text { currency (base } \\
\text { year) }\end{array}$ & $\begin{array}{l}\text { Included cost inputs and } \\
\text { assumptions ( } 1=\text { PPDC; } \\
2=\text { EDC })\end{array}$ & $\begin{array}{l}\text { Data quality } \\
\text { outcome link } \\
\text { with input } \\
\text { cost }\end{array}$ & $\begin{array}{l}\text { Cost range of inter- } \\
\text { vention }(1=\text { PPDC; } \\
2=E D C)\end{array}$ & Conclusions and remark \\
\hline $\begin{array}{l}\text { King et al } \\
{[7],} \\
\text { Ethiopia }\end{array}$ & $\begin{array}{l}\text { Input cost } \\
\text { analysis }\end{array}$ & $\begin{array}{ll}\bullet & 9433 \\
\bullet & 12,112\end{array}$ & $\begin{array}{ll}\bullet & \mathrm{NR}^{\mathrm{c}} \\
\text { - } & \mathrm{NR} \\
\bullet & \mathrm{US} \$ \\
& (2011)\end{array}$ & $\begin{array}{l}\text { - Printing and data } \\
\text { entry costs } \\
\text { Tablet and acces- } \\
\text { sories Single-use } \\
\text { cost }\end{array}$ & Not linked & $\begin{array}{l}\text { 1. US } \$ 13,883 \\
\text { 2. US } \$ 10,320\end{array}$ & $\begin{array}{l}\text { - Costs of the equip- } \\
\text { ment in EDC ap- } \\
\text { proximately the } \\
\text { same as with data } \\
\text { entry cost, recur- } \\
\text { rent use of EDC } \\
\text { may save costs. } \\
\text { Use of person day } \\
\text { for comparison of } \\
\text { personnel costs }\end{array}$ \\
\hline $\begin{array}{l}\text { McLean } \\
\text { et al [39], } \\
\text { Malawi }\end{array}$ & $\begin{array}{l}\text { Input cost } \\
\text { analysis } \\
\text { Differential } \\
\text { cost }\end{array}$ & $\begin{array}{ll}- & 426 \\
- & 558\end{array}$ & $\begin{array}{ll}- & 1 \text { year } \\
\text { - } & \text { NR } \\
\text { - } & \text { British } \\
& \text { Pound } \\
& (2016)\end{array}$ & $\begin{array}{l}\text { Printing and entry } \\
\text { cost and paper } \\
\text { archival } \\
\text { EDC development } \\
\text { and configuration, } \\
\text { device cost, data of- } \\
\text { ficer cost }\end{array}$ & Not linked & $\begin{array}{l}\text { 1. } £ 18,895 \\
\text { 2. } £ 11,427\end{array}$ & $\begin{array}{l}\text { In total, the esti- } \\
\text { mated costs for the } \\
\text { stages unique to } \\
\text { the paper-based } \\
\text { process is } 65 \% \\
\text { higher per annum } \\
\text { than the unique } \\
\text { costs for the EDC } \\
\text { system }\end{array}$ \\
\hline $\begin{array}{l}\text { Giduthuri } \\
\text { et al [37], } \\
\text { India }\end{array}$ & $\begin{array}{l}\text { Differential } \\
\text { input cost } \\
\text { analysis }\end{array}$ & $\begin{array}{l}98 \text { inter- } \\
\text { views for } \\
\text { both, and } \\
\text { extrapolated } \\
\text { for larger } \\
\text { samples } \\
1000\end{array}$ & $\begin{array}{ll}- & \mathrm{NR} \\
- & \mathrm{NR} \\
- & \mathrm{US} \$ \\
& (2013)\end{array}$ & $\begin{array}{l}\text { Printing and data } \\
\text { entry expenses } \\
\text { Cost of tablet com- } \\
\text { puters and server } \\
\text { charges }\end{array}$ & Not linked & $\begin{array}{l}\text { For } 96 \text { interviews, } \\
\text { the cost is } \\
\text { 1. US } \$ 2598 \\
\text { 2. US } \$ 2648\end{array}$ & $\begin{array}{l}\text { The initial invest- } \\
\text { ment in tablet- } \\
\text { based interviews } \\
\text { was higher com- } \\
\text { pared to paper, } \\
\text { while the recurring } \\
\text { costs per interview } \\
\text { were lower with } \\
\text { the use of tablets } \\
\text { EDC is less expen- } \\
\text { sive for larger sur- } \\
\text { veys }\end{array}$ \\
\hline $\begin{array}{l}\text { Mukasa } \\
\text { et al [38], } \\
\text { Tanzania }\end{array}$ & $\begin{array}{l}\text { Cost and } \\
\text { cost-effec- } \\
\text { tiveness }\end{array}$ & $\begin{array}{l}1000 \text { house- } \\
\text { holds for } \\
\text { both }\end{array}$ & $\begin{array}{l}\text { NR (used } \\
\text { deflator } \\
\text { values) } \\
\text { NR (used } \\
\text { the num- } \\
\text { ber of } \\
\text { house- } \\
\text { holds) } \\
\text { US \$ } \\
(2008)\end{array}$ & $\begin{array}{l}\text { - All fixed costs and } \\
\text { running costs } \\
\text { To estimate the } \\
\text { costs for } 2015 \text {, the } \\
\text { formula for expendi- } \\
\text { ture in } 2016=\mathrm{Ex}^{-} \\
\text {penditure in } 2008 \times \\
\text { Deflator } 2016, \text { defla- } \\
\text { tor } 2008 \\
\text { Deflator values of } \\
209.5 \text { for } 2008 \text { and } \\
233.6 \text { for } 2016\end{array}$ & Error rate & $\begin{array}{l}\text { For } 1000 \text { house- } \\
\text { holds, error-free } \\
\text { data set: } \\
\text { 1. US } \$ 1161 \\
\text { 2. US } \$ 9380 \\
\text { Crude data set: } \\
\text { 1. US } \$ 993 \\
\text { 2. US } \$ 891\end{array}$ & $\begin{array}{l}\text { For error-free data } \\
\text { sets, surveys using } \\
\text { electronic tools, } \\
\text { compared with pa- } \\
\text { per-based tools, } \\
\text { were less expen- } \\
\text { sive by } 28 \% \text { for } \\
\text { recurrent and } 19 \% \\
\text { for total costs }\end{array}$ \\
\hline
\end{tabular}




\begin{tabular}{|c|c|c|c|c|c|c|c|}
\hline $\begin{array}{l}\text { Study and } \\
\text { country }\end{array}$ & $\begin{array}{l}\text { Analytic } \\
\text { method or } \\
\text { model }\end{array}$ & $\begin{array}{l}\text { Interventions } \\
\text { studied or popula- } \\
\text { tion per group } \\
\left(1=\text { PPDC }^{\mathrm{a}}\right. \\
\left.2=\mathrm{EDC}^{\mathrm{b}}\right)\end{array}$ & $\begin{array}{l}\text { Time horizon, } \\
\text { discount rate, } \\
\text { currency (base } \\
\text { year) }\end{array}$ & $\begin{array}{l}\text { Included cost inputs and } \\
\text { assumptions ( } 1=\text { PPDC; } \\
2=\text { EDC })\end{array}$ & $\begin{array}{l}\text { Data quality } \\
\text { outcome link } \\
\text { with input } \\
\text { cost }\end{array}$ & $\begin{array}{l}\text { Cost range of inter- } \\
\text { vention }(1=\text { PPDC; } \\
2=\text { EDC })\end{array}$ & Conclusions and remark \\
\hline $\begin{array}{l}\text { Zhang et } \\
\text { al [42], } \\
\text { China }\end{array}$ & $\begin{array}{l}\text { Input cost } \\
\text { analysis }\end{array}$ & $\begin{array}{ll}- & 60 \\
- & 60 \\
- & \text { Extrapolat- } \\
\text { ed to } \\
\text { - } 1200 \\
\text { - } 1600\end{array}$ & $\begin{array}{ll}\text { - } & \text { NR } \\
\text { - } & \text { NR (used } \\
\text { projected } \\
\text { - } \\
\text { - Uamples) } \\
\quad(2012)\end{array}$ & $\begin{array}{l}\text { Both EDC and } \\
\text { PPDC: Items for } \\
\text { preparation, train- } \\
\text { ing, fieldwork and } \\
\text { data collection, and } \\
\text { logistics } \\
\text { Only printing and } \\
\text { transporting the } \\
\text { questionnaire, sta- } \\
\text { tionery, and data } \\
\text { entry } \\
\text { Only renting the } \\
\text { smartphone and the } \\
\text { software } \\
\text { Assumptions for the } \\
\text { extrapolation are } \\
\text { not known }\end{array}$ & Not linked & $\begin{array}{l}\text { Sample size: } 60 \\
\text { each } \\
\text { 1. US } \$ 1500 \\
\text { 2. US } \$ 2700 \\
\text { Sample size: } 1200 \\
\text { 1. US } \$ 41,570 \\
\text { 2. Cost per sam- } \\
\quad \text { ple, US } \\
\quad \$ 23.77 \\
\text { Sample size: } 1600 \\
\text { 1. US } \$ 28,520 \\
\text { 2. Cost per sam- } \\
\text { ple, US } \\
\quad \$ 25.98\end{array}$ & $\begin{array}{l}\text { - The mean costs } \\
\text { per questionnaire } \\
\text { were higher for } \\
\text { the smartphone } \\
\text { questionnaire } \\
\text { - No linked analysis } \\
\text { for data quality } \\
\text { and cost }\end{array}$ \\
\hline $\begin{array}{l}\text { Njuguna } \\
\text { et al [36], } \\
\text { Kenya }\end{array}$ & $\begin{array}{l}\text { Input cost } \\
\text { analysis }\end{array}$ & $\begin{array}{l}\text { - } \quad \text { Both } 880 \\
\text { Extrapolat- } \\
\text { ed for the } \\
\text { first year's } \\
\text { establishing } \\
\text { and second } \\
\text { year's oper- } \\
\text { ating cost }\end{array}$ & $\begin{array}{ll}\bullet & \text { NR } \\
\bullet & 2 \text { years } \\
\bullet & \text { US \$ } \\
& (2011, \\
2012)\end{array}$ & $\begin{array}{l}\text { First- and second- } \\
\text { year costs of start- } \\
\text { ing up and operating } \\
\text { based on payroll in- } \\
\text { formation }\end{array}$ & Not linked & $\begin{array}{l}\text { First year: } \\
\text { 1. US } \$ 15,999 \\
\text { 2. US } \$ 17,500 \\
\text { Second year: } \\
\text { 1. US } \$ 54,001 \\
\text { 2. US } \$ 50,200\end{array}$ & $\begin{array}{l}\text { For establishment } \\
\text { cost, EDC } 9.4 \% \\
\text { more than PPDC; } \\
\text { in } 2 \text { years, EDC } \\
\text { costs reduced } 7 \% \\
\text { compared to } \\
\text { PPDC } \\
\text { No linked analysis } \\
\text { for data quality } \\
\text { and cost }\end{array}$ \\
\hline $\begin{array}{l}\text { Dillon et } \\
\text { al [41], } \\
\text { South } \\
\text { Africa }\end{array}$ & $\begin{array}{l}\text { Input and } \\
\text { economic } \\
\text { analysis }\end{array}$ & $\begin{array}{ll}\text { - } & 95 \\
\bullet & 105\end{array}$ & $\begin{array}{l}\text { - } \quad \text { British } \\
\text { pound } \\
(2012)\end{array}$ & $\begin{array}{l}\text { - } \\
\text { - } \quad \text { Talary per correctly } \\
\text { Technology costs } \\
\text { and tablet computer } \\
\text { and additional over- } \\
\text { head costs (storage } \\
\text { space for paper hard } \\
\text { copies; office space } \\
\text { for data entry clerk, } \\
\text { and for EDC, hard- } \\
\text { ware maintenance } \\
\text { and upkeep) } \\
\text { Formulas presented } \\
\text { by Walther et al } \\
\text { [19]. } \\
\text { Assumed minimum } \\
\text { staffing: } \\
1 \text { field worker, } 1 \text { da- } \\
\text { ta entry clerk, and } 1 \\
\text { data supervisor } \\
1 \text { field worker and } 1 \\
\text { data manager } \\
\text { (EDC) } \\
\text { Calculation based } \\
\text { on } 46 \text { questions in a } \\
\text { questionnaire, } 5 \text { in- } \\
\text { terviews per day, } 22 \\
\text { working days per } \\
\text { month, } 110 \text { inter- } \\
\text { views per month, } \\
\text { and } 5060 \text { questions } \\
\text { per month } \\
\text { - }\end{array}$ & Error rate & $\begin{array}{l}\text { Salary cost per } \\
\text { month: } \\
\text { 1. } \quad £ 1000 \\
\text { 2. } £ 915 \\
\text { Equipment cost: } \\
\text { 1. } £ 420 \\
\text { 2. } \quad £ 1036\end{array}$ & 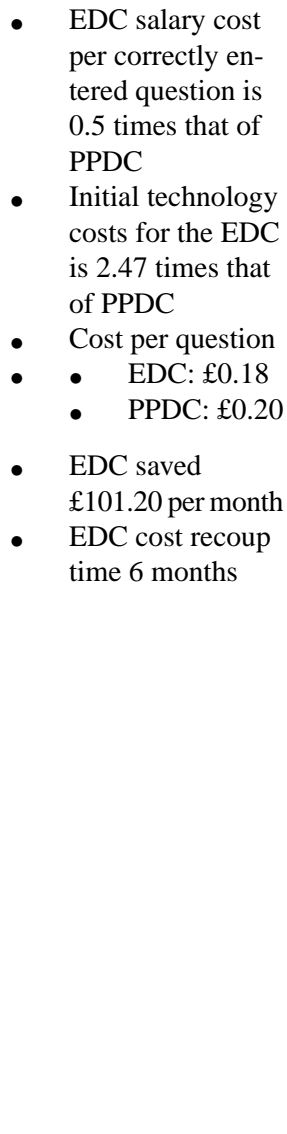 \\
\hline
\end{tabular}




\begin{tabular}{|c|c|c|c|c|c|c|c|}
\hline $\begin{array}{l}\text { Study and } \\
\text { country }\end{array}$ & $\begin{array}{l}\text { Analytic } \\
\text { method or } \\
\text { model }\end{array}$ & $\begin{array}{l}\text { Interventions } \\
\text { studied or popula- } \\
\text { tion per group } \\
\left(1=\text { PPDC }^{\text {a. }}\right. \\
\left.2=\text { EDC }^{\mathrm{b}}\right)\end{array}$ & $\begin{array}{l}\text { Time horizon, } \\
\text { discount rate, } \\
\text { currency (base } \\
\text { year) }\end{array}$ & $\begin{array}{l}\text { Included cost inputs and } \\
\text { assumptions ( } 1=\text { PPDC; } \\
2=\text { EDC) }\end{array}$ & $\begin{array}{l}\text { Data quality } \\
\text { outcome link } \\
\text { with input } \\
\text { cost }\end{array}$ & $\begin{array}{l}\text { Cost range of inter- } \\
\text { vention }(1=\text { PPDC; } \\
2=\text { EDC })\end{array}$ & Conclusions and remark \\
\hline $\begin{array}{l}\text { Flexman } \\
\text { et al [34], } \\
\text { Bangladesh } \\
\text { and } \\
\text { Philip- } \\
\text { pines }\end{array}$ & $\begin{array}{l}\text { Input data } \\
\text { analysis }\end{array}$ & $\begin{array}{ll}\text { - } & 5398 \\
\text { - } & 516\end{array}$ & $\begin{array}{l}2013 \text { ver- } \\
\text { bal autop- } \\
\text { sies' data } \\
\text { collec- } \\
\text { tion in } \\
\text { demo- } \\
\text { graphic } \\
\text { and } \\
\text { surveil- } \\
\text { lance } \\
\text { sites }\end{array}$ & $\begin{array}{l}\text { Differential cost es- } \\
\text { timation formula } \\
\text { excluding similar } \\
\text { cost for EDC and } \\
\text { PPDC, for example, } \\
\text { data collector cost } \\
\text { (see the full paper } \\
\text { mentioned on page } \\
\text { 4) } \\
\text { The cost for data } \\
\text { entry for a single } \\
\text { enterer is the one- } \\
\text { time cost of a com- } \\
\text { puter plus the } \\
\text { monthly salary mul- } \\
\text { tiplied by the num- } \\
\text { ber of months re- } \\
\text { quired }\end{array}$ & Not linked & $\begin{array}{l}\text { Printing cost } \\
\text { per paper } \\
\text { questionnaire } \\
\text { US \$0.246 } \\
\text { (Bangladesh) } \\
\text { and US } \\
\text { \$0.774 } \\
\text { (Philippines) } \\
\text { Cost of a sin- } \\
\text { gle electronic } \\
\text { tablet US } \\
\text { \$393.78 } \\
\text { (Bangladesh) } \\
\text { and US } \\
\text { \$365.76 } \\
\text { (Philippines) } \\
\text { Cost of a } \\
\text { computer for } \\
\text { data entry US } \\
\text { \$984.49 } \\
\text { (Bangladesh) } \\
\text { and US } \\
\text { \$1000.00 } \\
\text { (Philippines) } \\
\text { Monthly } \\
\text { salary of data } \\
\text { enterer US } \\
\text { \$384.62 } \\
\text { (Bangladesh) } \\
\text { and US } \\
\text { \$326.00 } \\
\text { (Philippines) } \\
\text { Average num- } \\
\text { ber of sam- } \\
\text { ples per per- } \\
\text { son per month } \\
107.8 \\
\text { (Bangladesh) } \\
\text { and 145.7 } \\
\text { (Philippines) }\end{array}$ & $\begin{array}{l}\text { For small-scale } \\
\text { surveys, the up- } \\
\text { front costs of pur- } \\
\text { chasing electronic } \\
\text { tablets was the pri- } \\
\text { mary cost, and it } \\
\text { had a higher total } \\
\text { cost. For large- } \\
\text { scale surveys, the } \\
\text { costs associated } \\
\text { with data entry ex- } \\
\text { ceeded the cost of } \\
\text { the tablets, so } \\
\text { EDC provides a } \\
\text { cheaper method of } \\
\text { data collection } \\
\text { Historical cost da- } \\
\text { ta from } 2 \text { coun- } \\
\text { tries. For projects } \\
\text { that require fewer } \\
\text { than } 150 \text { tablets } \\
\text { and collect over } \\
\text { 10,000 surveys, } \\
\text { the upfront cost of } \\
\text { the tablets will } \\
\text { likely be substan- } \\
\text { tially less than the } \\
\text { cost of data entry }\end{array}$ \\
\hline $\begin{array}{l}\text { Lietz et al } \\
{[40],} \\
\text { Burkina } \\
\text { Faso }\end{array}$ & $\begin{array}{l}\text { Compara- } \\
\text { tive input } \\
\text { cost analy- } \\
\text { sis }\end{array}$ & $\begin{array}{l}10,000 \\
\text { households } \\
\text { for both }\end{array}$ & 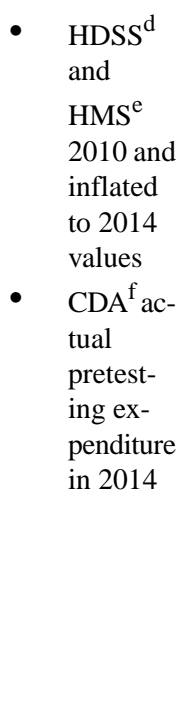 & $\begin{array}{l}\text { Fixed costs: person- } \\
\text { nel (team lead), of- } \\
\text { fice, and housekeep- } \\
\text { ing } \\
\text { Variable costs: data } \\
\text { collectors, supervi- } \\
\text { sors, consumables, } \\
\text { transportation, and } \\
\text { training (full text in } \\
\text { Textbox 1) } \\
\text { Fixed and variable } \\
\text { costs Financial costs } \\
\text { of the standalone } \\
\text { (HDSS and HMS) } \\
\text { and integrated } \\
\text { (CDA) survey ap- } \\
\text { proaches were esti- } \\
\text { mated from the per- } \\
\text { spective of the im- } \\
\text { plementing agency }\end{array}$ & Not assessed & $\begin{array}{l}\begin{array}{l}\text { Cost per household } \\
\text { visit } \\
\text { 1. }\end{array} \text { HMS + } \\
\text { HDSS } \\
\text { (PPDC): US } \\
\quad \text { \$251,641 } \\
\text { 2. } \quad \text { CDA (EDC): } \\
\text { US \$206,937 } \\
\text { Cost per (10,000 } \\
\text { HDSS) } \\
\text { 1. EDC: US \$21 } \\
\text { 2. PPDC: US } \\
\quad \text { \$25 }\end{array}$ & $\begin{array}{l}\text { - Cost analysis esti- } \\
\text { mated that the } \\
\text { CDA survey } \\
\text { would reduce the } \\
\text { annual costs of } \\
\text { survey implemen- } \\
\text { tation by about US } \\
\$ 45,000 \\
\text { No link with the } \\
\text { data quality out- } \\
\text { come }\end{array}$ \\
\hline
\end{tabular}


${ }^{\mathrm{a}} \mathrm{PPDC}$ : pen and paper data collection.

${ }^{b}$ EDC: electronic data collection.

${ }^{\mathrm{c}} \mathrm{NR}$ : not reported

${ }^{\mathrm{d}}$ HDSS: health and demographic surveillance systems.

${ }^{\mathrm{e}} \mathrm{HMS}$ : Household Morbidity Survey.

${ }^{\mathrm{f}} \mathrm{CDA}$ : Comprehensive Disease Assessment.

Studies from Ethiopia [7], Malawi [39], India [37], and Bangladesh and Philippines [34] reported a differential input cost unique to paper-based or electronic tools. For paper-based data collection, these were printing and data entry costs, and for EDC systems, the cost of electronic devices' hardware, software, and accessories. The general assumption was that all other costs, such as personnel costs, were the same for both tools. Another cost assumption was that of the cost of small-scale, short-duration surveys. Such kinds of small costs were extrapolated to large-scale surveys with no clear information about the model or the cost assumptions followed to reach the large-scale costs. The studies concluded that EDC was expensive for small-scale surveys, as the initial investment in hardware and software outweighs the paper-based printing and data entry costs. However, large-scale surveys showed a significant decrease in cost for EDC surveys; for example, the paper-based survey cost was up to $65 \%$ higher per annum than the unique costs for the EDC system [39]. None of these studies linked the input cost with data quality errors.

Detailed cost information and the link between cost and data quality were reported in a retrospective data analysis in Tanzania. However, the base year for the cost was 2008, and deflator values of 209.5 for 2008 and 233.6 for 2016 were used to report the costs. For 1000 households, the cost per error-free data set was US \$11,610 for PPDC and US \$9380 for EDC. For error-free data sets, surveys using electronic tools-compared with paper-based tools-were $28 \%$ less expensive in recurrent costs and $19 \%$ less expensive in total costs [38].

A study in South Africa also reported cost inputs linked to data quality outcomes [41]. The formula presented by Walther et al [19] was used with minimum staffing: for PPDC, 1 field worker, 1 data entry clerk, and 1 data supervisor, and for EDC, 1 field worker and 1 data manager. In addition, 46 questions in a questionnaire, 5 interviews per day, 22 working days per month, 110 interviews per month, and 5060 questions per month were planned. The EDC salary cost per correctly entered question was 0.5 times that of PPDC. Overall, the cost per question was $£ 0.18$ for $\mathrm{EDC}$ and $£ 0.20$ for PPDC. The equipment cost for PPDC was $£ 420$, compared with $£ 1036$ for EDC. EDC saved $£ 101.20$ per month, and the EDC cost recoup time was reported as 6 months.

Lingani et al [40] in Burkina Faso and Njuguna et al [36] in Kenya reported a detailed financial cost comparison for the establishment of PPDC and EDC at HDSS and a hospital-based surveillance system, respectively. The Kenyan report indicated that during establishment, the cost of EDC was 9.4\% higher than that of PPDC. However, after 2 years, EDC costs decreased by $7 \%$, compared with PPDC (see Table 4 for detailed cost information).

\section{Technology Characteristics, User Preference, and Acceptance}

The mobile devices used for data collection included PDAs, smartphones, tablet computers, and notebooks (Table 5). The included studies $(6 / 12,50 \%)$ also reported the use of open source Android apps called open data kit apps to customize the software according to their needs. Microsoft Windows and BlackBerry operating systems were installed on mobile devices.

Data transfer from mobile devices to the central server was conducted using one of the following methods:

- Direct transfer from the data collection site to the server using a mobile data network [34-36] and secure virtual private network [45].

- $\quad$ Direct transfer using Wi-Fi connection only [37,39].

- Transfer using secure digital memory card $[7,38]$.

- Transfer using USB cable [41].

Data transfer to a server located in a foreign country using a mobile network was not considered appropriate in some studies due to data ownership or security concerns $[7,43]$. 
Table 5. Extracted intervention or technology characteristics

\begin{tabular}{|c|c|c|c|c|c|}
\hline $\begin{array}{l}\text { Study and } \\
\text { country }\end{array}$ & $\begin{array}{l}\text { Analytic method or } \\
\text { model }\end{array}$ & $\begin{array}{l}\text { Interventions } \\
\text { studied or } \\
\text { population } \\
\text { per group } \\
\left(1=\mathrm{PPDC}^{\mathrm{a}} \text {; }\right. \\
\left.2=\mathrm{EDC}^{\mathrm{b}}\right)\end{array}$ & $\begin{array}{l}\text { Time horizon, discount } \\
\text { rate, currency (base year) }\end{array}$ & $\begin{array}{l}\text { Included cost inputs and assumptions } \\
(1=\text { PPDC; } 2=\text { EDC })\end{array}$ & $\begin{array}{l}\text { Data quality outcome link } \\
\text { with input cost }\end{array}$ \\
\hline $\begin{array}{l}\text { Njuguna } \\
\text { et al [36], } \\
\text { Ethiopia }\end{array}$ & $\begin{array}{l}\text { - } \quad \text { HTC smartphone } \\
\text { Software: FAST } \\
\text { kit: Microsoft } \\
\text { - } \quad \text { Purchased }\end{array}$ & $\begin{array}{l}\text { Direct to } \\
\text { server with } \\
\text { mobile net- } \\
\text { work }\end{array}$ & $\begin{array}{l}\text { - Programmed checks } \\
\text { and restrictions } \\
\text { Error message notifi- } \\
\text { cation for inaccurate } \\
\text { data entry }\end{array}$ & $\begin{array}{l}\text { - Limited or poor internet network } \\
\text { - Occasional server communication } \\
\text { breakdowns } \\
\text { - Delayed data submission }\end{array}$ & $\begin{array}{l}\text { Data saved in the smart- } \\
\text { phone's memory and later } \\
\text { uploaded on to the server } \\
\text { from convenient places }\end{array}$ \\
\hline $\begin{array}{l}\text { Ahmed et } \\
\text { al [35], } \\
\text { Sudan }\end{array}$ & $\begin{array}{ll}\text { - } & \text { Samsung smart- } \\
\text { - } & \text { phone } \\
\text { - } & \text { Open source }\end{array}$ & $\begin{array}{l}\text { Mobile inter- } \\
\text { net network }\end{array}$ & - $\quad$ ODK functionalities & $\begin{array}{l}\text { - Limited or poor internet network in } \\
\text { certain areas } \\
\text { - Unexpected software closure or } \\
\text { freeze } \\
\text { - Accessibility of the aggregate web- } \\
\text { site in Sudan was challenging } \\
\text { - Using Arabic language in the ODK } \\
\text { was challenging for form develop- } \\
\text { ment and data retrieval }\end{array}$ & $\begin{array}{l}\text { Used ODK offline and } \\
\text { submitted the data after } \\
\text { restarting the smartphone }\end{array}$ \\
\hline $\begin{array}{l}\text { King et al } \\
{[7],} \\
\text { Ethiopia }\end{array}$ & $\begin{array}{ll}\text { - } & \text { Samsung tablet } \\
\text { - } & \text { Somputer } \\
& \text { Swift Insights Mo- } \\
\text { bile } 1.1 \\
\text { Barcode scanner } \\
\text { 4.3.1 Open source } \\
\text { developed }\end{array}$ & $\begin{array}{l}\mathrm{SD}^{\mathrm{c}} \text { card } \\
\text { with pass- } \\
\text { word-protect- } \\
\text { ed download- } \\
\text { ing to the su- } \\
\text { pervisor's } \\
\text { laptop }\end{array}$ & $\begin{array}{l}\text { ODK functionali- } \\
\text { ties+ User-defined } \\
\text { survey preferences, } \\
\text { generation of } \\
\text { unique record identi- } \\
\text { fication (Textbox 1) }\end{array}$ & $\begin{array}{l}\text { - Limited or poor internet network in } \\
\text { certain areas }\end{array}$ & $\begin{array}{l}\text { Data stored first on the su- } \\
\text { pervisor laptop and then } \\
\text { uploaded to the local serv- } \\
\text { er to maintain the } \\
\text { sovereignty and security of } \\
\text { the data set }\end{array}$ \\
\hline $\begin{array}{l}\text { Flexman } \\
\text { et al [34], } \\
\text { Bangladesh } \\
\text { and } \\
\text { Philip- } \\
\text { pines }\end{array}$ & $\begin{array}{ll}- & \text { Samsung tablets } \\
\text { - } & \text { ODK } \\
\text { - } & \text { Customized in } \\
& \text { ODK }\end{array}$ & $\begin{array}{l}\text { Direct to } \\
\text { server with } \\
\text { mobile net- } \\
\text { work }\end{array}$ & $\begin{array}{l}\text { ODK functionalities } \\
\text { (no explicit descrip- } \\
\text { tion) }\end{array}$ & - $\quad$ Sufficiently strong internet network & $\begin{array}{l}\text { Purchase memory cards for } \\
\text { the tablets to back up data } \\
\text { locally }\end{array}$ \\
\hline $\begin{array}{l}\text { Giduthuri } \\
\text { et al [37], } \\
\text { India }\end{array}$ & 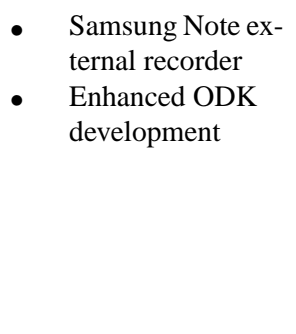 & $\begin{array}{l}\text { Encrypted } \\
\text { and upload- } \\
\text { ed over a } \\
\text { Wi-Fi con- } \\
\text { nection to a } \\
\text { central serv- } \\
\text { er after re- } \\
\text { turning to } \\
\text { the office }\end{array}$ & Not described & $\begin{array}{l}\text { - Required highly trained interview- } \\
\text { ers } \\
\text { Data loss by the interviewer due to } \\
\text { accidentally pressing the delete } \\
\text { button }\end{array}$ & Not described \\
\hline $\begin{array}{l}\text { Musaka } \\
\text { et al [38], } \\
\text { Tanzania }\end{array}$ & $\begin{array}{ll}\text { - } & \text { PDA BlackBerry } \\
\text { - } & \text { HRS }^{\mathrm{d}} \text { software } \\
\text { - } & \text { BlackBerry OS } \\
\text { - } & \text { Customized in } \\
& \text { HRS software }\end{array}$ & $\begin{array}{l}\text { Micro SD } \\
\text { card }\end{array}$ & - $\quad$ Skip function & $\begin{array}{l}\text { - Device stopped functioning during } \\
\text { interview or submission }\end{array}$ & Not described \\
\hline $\begin{array}{l}\text { McLean } \\
\text { et al [39], } \\
\text { Malawi }\end{array}$ & $\begin{array}{ll}\text { - } & \text { Toshiba tablets } \\
\text { and Samsung } \\
\text { smartphones } \\
\text { - } & \text { ODK } \\
\text { - } & \text { Contractual pay- } \\
\text { ment for form de- } \\
\text { velopment }\end{array}$ & $\begin{array}{l}\text { Secured } \\
\text { wireless net- } \\
\text { work (not re- } \\
\text { layed on } \\
\text { phone net- } \\
\text { work) }\end{array}$ & - $\quad$ ODK functionalities & $\begin{array}{l}\text { - The existing wireless network was } \\
\text { sufficiently strong to upload the } \\
\text { data } \\
\text { - } \quad \text { Adequate battery life for a day } \\
\text { - } \quad \text { Lack of internal staff for form devel- } \\
\text { opment } \\
\text { - } \quad \text { Required outsourcing cost } \\
11 \text { of } 92 \text { tablets broke in } 4 \text { years and } \\
\text { had to be replaced } \\
\text { Only } 1 \text { functioning tablet and } 1 \\
\text { smartphone went missing over the } \\
\text { 4-year period }\end{array}$ & $\begin{array}{l}\text { A dedicated, secured de- } \\
\text { vice charging area }\end{array}$ \\
\hline
\end{tabular}




\begin{tabular}{|c|c|c|c|c|c|}
\hline $\begin{array}{l}\text { Study and } \\
\text { country }\end{array}$ & $\begin{array}{l}\text { Analytic method or } \\
\text { model }\end{array}$ & $\begin{array}{l}\text { Interventions } \\
\text { studied or } \\
\text { population } \\
\text { per group } \\
\left(1=\text { PPDC }^{\mathrm{a}} \text {; }\right. \\
\left.2=\mathrm{EDC}^{\mathrm{b}}\right)\end{array}$ & $\begin{array}{l}\text { Time horizon, discount } \\
\text { rate, currency (base year) }\end{array}$ & $\begin{array}{l}\text { Included cost inputs and assumptions } \\
(1=\text { PPDC; } 2=\text { EDC })\end{array}$ & $\begin{array}{l}\text { Data quality outcome link } \\
\text { with input cost }\end{array}$ \\
\hline $\begin{array}{l}\text { Dillon et } \\
\text { al [41], } \\
\text { South } \\
\text { Africa }\end{array}$ & $\begin{array}{l}\text { - Tablet, PC, and } \\
\text { mobile phone } \\
\text { - Self-developed a } \\
\text { C\#-based program } \\
\text { with XML }\end{array}$ & $\begin{array}{l}\text { Data transfer } \\
\text { through USB } \\
\text { connections, } \\
\text { avoiding the } \\
\text { need for a } \\
\text { constant in- } \\
\text { ternet con- } \\
\text { nection }\end{array}$ & $\begin{array}{l}\text { Facilitate data } \\
\text { checks and early de- } \\
\text { tection and correc- } \\
\text { tion of faulty proce- } \\
\text { dures and data man- } \\
\text { agement }\end{array}$ & 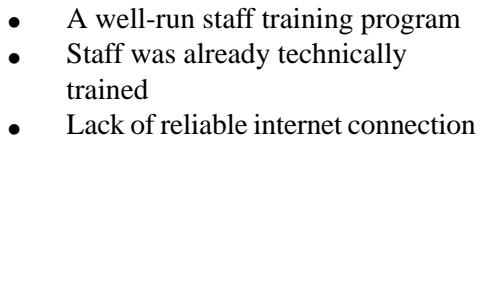 & Data transfer through USB \\
\hline $\begin{array}{l}\text { Byass et } \\
\text { al [44], } \\
\text { Burkina } \\
\text { Faso }\end{array}$ & $\begin{array}{l}-\quad \text { PDA } \\
\text { - } \quad \text { Pendragon } 4 \text { soft- } \\
\text { ware programmed }\end{array}$ & $\begin{array}{l}\text { Data copied } \\
\text { for PDAs' } \\
\text { memory } \\
\text { cards }\end{array}$ & - $\quad$ GPS, time stamp & $\begin{array}{l}\text { Use several available mobile- } \\
\text { charging arrangements in vehicles } \\
\text { or solar panels } \\
\text { - } \quad \text { Temporary system clock changes } \\
\text { in a few PDAs } \\
\text { - } \quad \text { Some incomplete GPS data strings } \\
\text { - } 48 / 151 \text { PDA encountered technical } \\
\text { errors }\end{array}$ & $\begin{array}{l}\text { Saving data from the } \\
\text { PDAs' internal volatile } \\
\text { memory to nonvolatile } \\
\text { memory cards. Protective } \\
\text { plastic cover for the safety } \\
\text { of PDA was important }\end{array}$ \\
\hline $\begin{array}{l}\text { Maduga } \\
\text { et al [46], } \\
\text { Nigeria }\end{array}$ & $\begin{array}{ll}\text { - } & \text { Mobile phone } \\
\text { - } & \text { ODK } \\
\text { - } & \text { Android } \\
& \text { Locally cus- } \\
& \text { tomized }\end{array}$ & $\begin{array}{l}\text { Trained to } \\
\text { upload com- } \\
\text { pleted forms } \\
\text { onto a secure } \\
\text { server, with } \\
\text { back-end ac- } \\
\text { cess provid- } \\
\text { ed to only } \\
\text { the research } \\
\text { team lead }\end{array}$ & - $\quad$ ODK functionalities & $\begin{array}{ll}\text { - } & \text { Limited network connection } \\
\text { - } & \text { Fluctuation of power and internet } \\
\text { connection }\end{array}$ & $\begin{array}{l}\text { The extra } 2 \text { phones served } \\
\text { as backup in the event of } \\
\text { malfunction or challenges } \\
\text { with the global system for } \\
\text { mobile communication } \\
\text { mobile and data networks. } \\
\text { Multiple SIM cards were } \\
\text { provided in an attempt to } \\
\text { mitigate the problem }\end{array}$ \\
\hline
\end{tabular}

\footnotetext{
${ }^{\mathrm{a}}$ FAST: Field Adapted Survey Toolkit.

bODK: Open Data Kit.

${ }^{\mathrm{c}} \mathrm{SD}$ : Secure Digital.

${ }^{\mathrm{d}}$ HRS: Household Registration System.

${ }^{\mathrm{e}} \mathrm{OS}$ : operating system.
}

\section{Technical Challenges in Electronic Data Collection}

Limited or poor internet connectivity, occasional server communication interruptions, language challenges, the need for highly trained data collectors, device stack or freezing, device loss and breakdowns, and limited battery or power sources are among the technological challenges faced in the implementation of EDC systems (Table 5). The solutions include offline storage and transfer as soon as the data collectors obtain reliable internet connection (store and forward methods), transferring data using USB cables or secure digital cards, purchasing backup mobile devices or batteries, and using paper questionnaires at times of device malfunction.

\section{Preference, Acceptability, and Feelings}

A total of 6 papers [7,34,37-39,42] reported that user preferences, acceptance, and opinions were assessed using formal evaluation methods, such as individual or focus groups or qualitative interviews. Detailed comparative advantages and disadvantages of paper and electronics tools are reported in thematic-based tables in [7,38,39].

The use of smartphones to collect data was faster, easier to follow, and more convenient, as the data collectors did not have to carry cumbersome paper questionnaires and less space was needed to store their data collection tools. Additional functionalities — such as automatic retrieval of respondents and 
other members of the household or GPS functionalities-are also reported as an advantage.

The risk of data loss with paper-based surveys was perceived as being less than that with EDC, as paper questionnaires are tangible and enable immediate review, identification, and correction of mistakes. Paper surveys were also perceived to be easier for manipulating, adding, or changing data - for instance, including a household member absent during the survey who was later encountered by the survey team. The automated skip function was advantageous and time saving. The enumerators did not have to read the questions on every visit to the same household.

Enumerators described that the devices felt exciting, interesting, and prestigious, and they were skilled professionals in the eyes of the community. Some fieldworkers felt that EDC interviews took longer, and occasionally, devices froze during an interview.

Some studies that were excluded from our review also offer important insights about preference, acceptability, and local experiences [48-51].

\section{Discussion}

This systematic review synthesized the available comparative evidence on paper-based and electronic data collection tools and the potential effect of using these tools on data quality, implementation cost, and user preferences in interview-administered public health surveys. The systematic review included studies from 2008 to 2018 that were identified through multiple online electronic database searches. We identified more than 3500 papers and screened more than 2500 titles and abstracts to include 14 full-text papers based on our inclusion criteria. We extracted and synthesized available evidence regarding data quality, cost-effectiveness, timeliness, and user preferences. No paper has reported a study design with a classical randomized control approach. Randomization was reported to indicate either respondent allocation to paper-based or electronic tools or data collectors' exposure to one of the data collection tools. Meta-analysis was not possible due to the heterogeneous nature of study designs, measurements and outcome types, and study settings. Instead, a narrative synthesis based on predefined data quality, cost and related outcomes' acceptability, and preferences was conducted.

We employed a rigorous systematic review process to formulate the research questions, prepare individual database-tailored search strategies, execute searches in multiple databases, and independently screen and extract evidence from thousands of papers.

However, the results were inadequate for meta-analysis. The final included studies were heterogeneous and could not be combined to generate better estimates. Low-quantity and low-quality phenomena are becoming evident in most recent systematic reviews assessing mHealth or eHealth outcomes $[13,29,30]$

This scarcity might result from the following reasons: lack of primary studies with a rigorous study design, insufficient search strategy or review process, or unnecessary narrowing of a study focus. The most commonly reported reason is a lack of sufficient, well-planned, rigorous studies. The lack of evidence might be due to reluctance to evaluate the system after implementation and due to publication bias because of unsuccessful or disappointing findings [52]. Apart from a few studies $[35,37,39,41,42]$, those included in our systematic review were not primarily designed to evaluate the impact of EDC compared with PPDC. Instead, the studies were a byproduct of survey experience from a comparative analysis using their secondary data. Information from such implementation practices can provide insights or lessons for readers, but the comparative outcomes might be influenced by unplanned and uncontrolled confounding variables [7,36]. Elimination of observed and unobserved factors that might otherwise plausibly explain the difference in outcomes in the study design can increase confidence in the assertion that the estimated impact constitutes the real impact of the tools. The studies included in this review suffered from multiple biases during sample size estimation, selection (purposive vs random selection), and data quality outcome measurement level (before or after data entry). We recommend that future research focus specifically on the mode of data collection measurement and on quantifying the impacts with sound research designs.

Generating a full economic evaluation of the evidence facilitates a comparison between interventions in terms of their costs and intended outcomes and can be used to inform decisionmakers or funders of the available choices among alternatives upon cost justification $[53,54]$. In our systematic review, we attempted to extract the available cost information using the CHEERS checklist [47]; however, most of the expected items in the checklist were not reported. A majority of the studies lacked a detailed description of unit costs, data sources, and cost calculations. Moreover, most used a time horizon of 1 year and failed to assess long-term costs and data quality effects. The rationale for the choice of the time horizon was also not explicitly stated.

Despite these limitations, the available cost data could provide clues regarding the existing cost parameters for paper- and electronic data collection systems. Two studies managed to link the cost of implementing EDC and PPDC tools to data quality. Such cost-effectiveness analyses should be encouraged in future studies. There is no clear answer or guideline to shape the type and level of rigorous studies in health information technology evaluation [55,56]. Details of evidence-based health informatics history, current practices, and future recommendations are discussed elsewhere [52]. Further debate and consensus among academics and researchers in biomedical informatics should continue to determine how and when health information technology evaluation is rigorous and produces good quality data [57].

The studies identified in this review were conducted in various countries and in the context of different health care systems. Generalizing and applying results from different contexts is difficult because of variations in clinical practice, costs, and their analysis. However, what was consistent across all studies was a lack of reporting on the feasibility of adopting these technologies based on economic and organizational factors. 
It was surprising to see limited publications from global survey implementation organizations for the DHS and INDEPTH network groups, as those projects have many years of multinational implementation experience [4,58]; however, apart from experience reports, comparative evaluation studies in those areas are rare. Further evaluation research in these projects might produce evidence of data quality, cost, timeliness, and the success and failure factors for multinational projects.

There are positive perceptions regarding the acceptability, usability, and preference of EDC over PPDC among data collectors. This positivity is because technology enables data collectors to focus on their work, get immediate feedback regarding their mistakes, correct their errors while in the field, and leave few data quality issues to revisit. It is not known whether this excitement is a short-term effect immediately after the technology introduction or a stable long-term view based on longer exposure. A short period of technophilic or technophobic attitudes might lead to inaccurate overall impression of the tool, as accurate impression can only develop with longer exposure to the technology [59]. The generalizability and applicability of the results, given the different types of devices with different technical specifications and the rapid pace at which technology advances, need critical evaluation.
The generalizability of the findings of this systematic review is also challenged by the studies themselves, considering the variations in the characteristics of data collectors, level of outcome measurements, settings of the survey, and the psychometric properties of the survey questionnaires.

\section{Conclusions}

This systematic review showed that, despite consistent claims of a positive impact of technology on data quality and cost-effectiveness, the available evidence is small in quantity and low in quality. Purposefully designed comparative studies assessing the impact of data quality and cost-effectiveness are needed for implementation in organizations and by decision makers.

Despite the heterogeneity and low quality of the included studies, their qualitative synthesis showed the superiority of EDC systems over paper-based systems for data quality, process efficiencies, and cost.

Comparative evaluation studies sourced from international survey-implementing organizations where their routine data collection mode is EDC can provide a better platform for impact evaluation research in large-scale surveys.

\section{Acknowledgments}

The authors would like to thank the Deutscher Akademischer Austauschdienst for funding the research stay of the principal investigator.

\section{Conflicts of Interest}

None declared.

\section{Multimedia Appendix 1}

Additional search strategies.

[DOCX File, 17 KB-Multimedia Appendix 1]

\section{References}

1. Bocquier P, Sankoh O, Byass P. Are health and demographic surveillance system estimates sufficiently generalisable? Glob Health Action 2017;10(1):1356621 [FREE Full text] [doi: 10.1080/16549716.2017.1356621] [Medline: 28820344]

2. Timaeus I, Harpham T, Price M, Gilson L. Health surveys in developing countries: the objectives and design of an international programme. Soc Sci Med 1988;27(4):359-368. [doi: 10.1016/0277-9536(88)90270-5] [Medline: 3175718]

3. Gerritsen A, Bocquier P, White M, Mbacké C, Alam N, Beguy D, et al. Health and demographic surveillance systems: contributing to an understanding of the dynamics in migration and health. Glob Health Action 2013 Jul 11;6(1):21496 [FREE Full text] [doi: 10.3402/gha.v6i0.21496] [Medline: 23849188]

4. Survey Organization Manual for Demographic and Health Surveys. ICF International. 2012. URL: https://dhsprogram.com/ pubs/pdf/DHSM10/DHS6 Survey Org Manual 7Dec2012 DHSM10.pdf [accessed 2018-12-05]

5. Ethiopia Demographic and Health Survey. Central Statistical Agency [Ethiopia] and ICF International. 2012. URL: https:/ /dhsprogram.com/countries/Country-Main.cfm?ctry id=65 [accessed 2018-10-02]

6. Okwaraji YB, Cousens S, Berhane Y, Mulholland K, Edmond K. Effect of geographical access to health facilities on child mortality in rural Ethiopia: a community based cross sectional study. PLoS One 2012;7(3):e33564 [FREE Full text] [doi: 10.1371/journal.pone.0033564] [Medline: 22428070]

7. King JD, Buolamwini J, Cromwell EA, Panfel A, Teferi T, Zerihun M, et al. A novel electronic data collection system for large-scale surveys of neglected tropical diseases. PLoS One 2013;8(9):e74570 [FREE Full text] [doi: 10.1371/journal.pone.0074570] [Medline: 24066147]

8. Toninelli D, Pinter R, Pablo DP. Mobile Research Methods: Opportunities and Challenges of Mobile Research Methodologies. London, UK: Ubiquity Press; 2015. 
9. Bowling A. Mode of questionnaire administration can have serious effects on data quality. J Public Health (Oxf) 2005 Sep;27(3):281-291. [doi: 10.1093/pubmed/fdi031] [Medline: $\underline{15870099]}$

10. Groves R. Survey Errors and Survey Costs. New Jersey: John Wiley \& Sons; 2004.

11. World Health Organization. Global Diffusion of Ehealth: Making Universal Health Coverage Achievable. Report of the Third Global Survey on Ehealth. Geneva, Switzerland: World Health Organization; 2016.

12. Labrique AB, Vasudevan L, Kochi E, Fabricant R, Mehl G. mHealth innovations as health system strengthening tools: 12 common applications and a visual framework. Glob Health Sci Pract 2013 Aug 6;1(2):160-171. [doi: 10.9745/ghsp-d-13-00031]

13. Braun R, Catalani C, Wimbush J, Israelski D. Community health workers and mobile technology: a systematic review of the literature. PLoS One 2013;8(6):e65772 [FREE Full text] [doi: 10.1371/journal.pone.0065772] [Medline: 23776544]

14. Marcano Belisario JS, Jamsek J, Huckvale K, O'Donoghue J, Morrison C, Car J. Comparison of self-administered survey questionnaire responses collected using mobile apps versus other methods. Cochrane Database Syst Rev 2015 Jul 27(7):MR000042. [doi: 10.1002/14651858.MR000042.pub2] [Medline: 26212714]

15. Perlis TE, Des Jarlais DC, Friedman SR, Arasteh K, Turner CF. Audio-computerized self-interviewing versus face-to-face interviewing for research data collection at drug abuse treatment programs. Addiction 2004 Jul;99(7):885-896. [doi: 10.1111/j.1360-0443.2004.00740.x] [Medline: 15200584]

16. Adebajo S, Obianwu O, Eluwa G, Vu L, Oginni A, Tun W, et al. Comparison of audio computer assisted self-interview and face-to-face interview methods in eliciting HIV-related risks among men who have sex with men and men who inject drugs in Nigeria. PLoS One 2014;9(1):e81981 [FREE Full text] [doi: 10.1371/journal.pone.0081981] [Medline: 24416134]

17. Beauclair R, Meng F, Deprez N, Temmerman M, Welte A, Hens N, et al. Evaluating audio computer assisted self-interviews in urban South African communities: evidence for good suitability and reduced social desirability bias of a cross-sectional survey on sexual behaviour. BMC Med Res Methodol 2013 Jan 31;13:11 [FREE Full text] [doi: 10.1186/1471-2288-13-11] [Medline: 23368888]

18. Richens J, Copas A, Sadiq ST, Kingori P, McCarthy O, Jones V, et al. A randomised controlled trial of computer-assisted interviewing in sexual health clinics. Sex Transm Infect 2010 Aug;86(4):310-314. [doi: 10.1136/sti.2010.043422] [Medline: 20551234]

19. Walther B, Hossin S, Townend J, Abernethy N, Parker D, Jeffries D. Comparison of electronic data capture (EDC) with the standard data capture method for clinical trial data. PLoS One 2011;6(9):e25348 [FREE Full text] [doi:

10.1371/journal.pone.0025348] [Medline: 21966505]

20. Le Jeannic A, Quelen C, Alberti C, Durand-Zaleski I, CompaRec Investigators. Comparison of two data collection processes in clinical studies: electronic and paper case report forms. BMC Med Res Methodol 2014 Jan 17;14:7 [FREE Full text] [doi: 10.1186/1471-2288-14-7] [Medline: 24438227]

21. Pavlović I, Kern T, Miklavcic D. Comparison of paper-based and electronic data collection process in clinical trials: costs simulation study. Contemp Clin Trials 2009 Jul;30(4):300-316. [doi: 10.1016/j.cct.2009.03.008] [Medline: 19345286]

22. Rorie DA, Flynn RW, Grieve K, Doney A, Mackenzie I, MacDonald TM, et al. Electronic case report forms and electronic data capture within clinical trials and pharmacoepidemiology. Br J Clin Pharmacol 2017 Sep;83(9):1880-1895 [FREE Full text] [doi: 10.1111/bcp.13285] [Medline: 28276585]

23. di Pasquale A, McCann RS, Maire N. Assessing the population coverage of a health demographic surveillance system using satellite imagery and crowd-sourcing. PLoS One 2017;12(8):e0183661 [FREE Full text] [doi: 10.1371/journal.pone.0183661] [Medline: 28859109]

24. Homan T, Di Pasquale A, Kiche I, Onoka K, Hiscox A, Mweresa C, et al. Innovative tools and OpenHDS for health and demographic surveillance on Rusinga Island, Kenya. BMC Res Notes 2015 Sep 1;8:397 [FREE Full text] [doi: 10.1186/s13104-015-1373-8] [Medline: 26323664]

25. Kaneko S, K'opiyo J, Kiche I, Wanyua S, Goto K, Tanaka J, et al. Health and demographic surveillance system in the western and coastal areas of Kenya: an infrastructure for epidemiologic studies in Africa. J Epidemiol 2012;22(3):276-285 [FREE Full text] [doi: 10.2188/jea.je20110078] [Medline: 22374366]

26. Sifuna P, Oyugi M, Ogutu B, Andagalu B, Otieno A, Owira V, et al. Int J Epidemiol 2014 Aug;43(4):1097-1104 [FREE Full text] [doi: 10.1093/ije/dyu139] [Medline: 25009309]

27. Kishamawe C, Isingo R, Mtenga B, Zaba B, Todd J, Clark B, et al. Int J Epidemiol 2015 Dec;44(6):1851-1861 [FREE Full text] [doi: 10.1093/ije/dyv188] [Medline: 26403815]

28. Okwaraji YB, Webb EL, Edmond KM. Barriers in physical access to maternal health services in rural Ethiopia. BMC Health Serv Res 2015 Nov 4;15:493 [FREE Full text] [doi: 10.1186/s12913-015-1161-0] [Medline: 26537884]

29. Iribarren SJ, Cato K, Falzon L, Stone PW. What is the economic evidence for mHealth? A systematic review of economic evaluations of mHealth solutions. PLoS One 2017;12(2):e0170581 [FREE Full text] [doi: 10.1371/journal.pone.0170581] [Medline: 28152012]

30. Agarwal S, Perry HB, Long L, Labrique AB. Evidence on feasibility and effective use of mHealth strategies by frontline health workers in developing countries: systematic review. Trop Med Int Health 2015 Aug;20(8):1003-1014 [FREE Full text] [doi: 10.1111/tmi.12525] [Medline: 25881735] 
31. Moher D, Liberati A, Tetzlaff J, Altman DG, PRISMA Group. Preferred reporting items for systematic reviews and meta-analyses: the PRISMA statement. PLoS Med 2009 Jul 21;6(7):e1000097 [FREE Full text] [doi: 10.1371/journal.pmed.1000097] [Medline: 19621072]

32. Liberati A, Altman DG, Tetzlaff J, Mulrow C, Gøtzsche PC, Ioannidis JP, et al. The PRISMA statement for reporting systematic reviews and meta-analyses of studies that evaluate health care interventions: explanation and elaboration. PLoS Med 2009 Jul 21;6(7):e1000100 [FREE Full text] [doi: 10.1371/journal.pmed.1000100] [Medline: 19621070]

33. Zeleke AA, Naziyok T, Fritz F, Röhrig R. Data quality and cost-effectiveness analyses of electronic and paper-based interviewer-administered public health surveys: protocol for a systematic review. JMIR Res Protoc 2019 Jan 30;8(1):e10678 [FREE Full text] [doi: 10.2196/10678] [Medline: $\underline{\text { 30698530] }}$

34. Flaxman AD, Stewart A, Joseph JC, Alam N, Alam SS, Chowdhury H, et al. Collecting verbal autopsies: improving and streamlining data collection processes using electronic tablets. Popul Health Metr 2018 Feb 1;16(1):3 [FREE Full text] [doi: 10.1186/s12963-018-0161-9] [Medline: 29391038]

35. Ahmed R, Robinson R, Elsony A, Thomson R, Squire SB, Malmborg R, et al. A comparison of smartphone and paper data-collection tools in the Burden of Obstructive Lung Disease (BOLD) study in Gezira state, Sudan. PLoS One 2018;13(3):e0193917 [FREE Full text] [doi: 10.1371/journal.pone.0193917] [Medline: 29518132]

36. Njuguna HN, Caselton DL, Arunga GO, Emukule GO, Kinyanjui DK, Kalani RM, et al. A comparison of smartphones to paper-based questionnaires for routine influenza sentinel surveillance, Kenya, 2011-2012. BMC Med Inform Decis Mak 2014 Dec 24;14:107 [FREE Full text] [doi: 10.1186/s12911-014-0107-5] [Medline: 25539745]

37. Giduthuri JG, Maire N, Joseph S, Kudale A, Schaetti C, Sundaram N, et al. Developing and validating a tablet version of an illness explanatory model interview for a public health survey in Pune, India. PLoS One 2014;9(9):e107374 [FREE Full text] [doi: 10.1371/journal.pone.0107374] [Medline: 25233212]

38. Mukasa O, Mushi HP, Maire N, Ross A, de Savigny D. Do surveys with paper and electronic devices differ in quality and cost? Experience from the Rufiji Health and demographic surveillance system in Tanzania. Glob Health Action 2017;10(1):1387984 [FREE Full text] [doi: 10.1080/16549716.2017.1387984] [Medline: 29157182]

39. McLean E, Dube A, Saul J, Branson K, Luhanga M, Mwiba O, et al. Implementing electronic data capture at a well-established health and demographic surveillance site in rural northern Malawi. Glob Health Action 2017;10(1):1367162 [FREE Full text] [doi: 10.1080/16549716.2017.1367162] [Medline: 28922071]

40. Lietz H, Lingani M, Sié A, Sauerborn R, Souares A, Tozan Y. Measuring population health: costs of alternative survey approaches in the Nouna Health and Demographic Surveillance System in rural Burkina Faso. Glob Health Action 2015;8:28330 [FREE Full text] [doi: 10.3402/gha.v8.28330] [Medline: 26257048]

41. Dillon DG, Pirie F, Rice S, Pomilla C, Sandhu MS, Motala AA, African Partnership for Chronic Disease Research (APCDR). Open-source electronic data capture system offered increased accuracy and cost-effectiveness compared with paper methods in Africa. J Clin Epidemiol 2014 Dec;67(12):1358-1363 [FREE Full text] [doi: 10.1016/j.jclinepi.2014.06.012] [Medline: 25135245]

42. Zhang S, Wu Q, van Velthoven MH, Chen L, Car J, Rudan I, et al. Smartphone versus pen-and-paper data collection of infant feeding practices in rural China. J Med Internet Res 2012 Sep 18;14(5):e119 [FREE Full text] [doi: 10.2196/jmir.2183] [Medline: 22989894]

43. Zhang J, Joshi R, Sun J, Rosenthal SR, Tong M, Li C, et al. A feasibility study on using smartphones to conduct short-version verbal autopsies in rural China. Popul Health Metr 2016;14:31 [FREE Full text] [doi: 10.1186/s12963-016-0100-6] [Medline: 27547126]

44. Byass P, Hounton S, Ouédraogo M, Somé H, Diallo I, Fottrell E, et al. Direct data capture using hand-held computers in rural Burkina Faso: experiences, benefits and lessons learnt. Trop Med Int Health 2008 Jul;13(Suppl 1):25-30 [FREE Full text] [doi: 10.1111/j.1365-3156.2008.02084.x] [Medline: 18578809]

45. Ojwang JK, Lee VC, Waruru A, Ssempijja V, Ng ang a JG, Wakhutu BE, et al. Using information and communications technology in a national population-based survey. J Acquired Immun Def Syndrome 2014;66:S123-S129. [doi: 10.1097/qai.0000000000000116]

46. Maduka O, Akpan G, Maleghemi S. Using android and open data kit technology in data management for research in resource-limited settings in the niger delta region of nigeria: cross-sectional household survey. JMIR Mhealth Uhealth 2017 Nov 30;5(11):e171 [FREE Full text] [doi: 10.2196/mhealth.7827] [Medline: 29191798]

47. Husereau D, Drummond M, Petrou S, Carswell C, Moher D, Greenberg D, CHEERS Task Force. Consolidated Health Economic Evaluation Reporting Standards (CHEERS) statement. Br Med J 2013 Mar 25;346:f1049 [FREE Full text] [doi: 10.1136/bmj.f1049] [Medline: 23529982]

48. Seebregts CJ, Zwarenstein M, Mathews C, Fairall L, Flisher AJ, Seebregts C, et al. Handheld computers for survey and trial data collection in resource-poor settings: development and evaluation of PDACT, a Palm Pilot interviewing system. Int J Med Inform 2009 Nov;78(11):721-731. [doi: 10.1016/j.ijmedinf.2008.10.006] [Medline: 19157967]

49. Paudel D, Ahmed M, Pradhan A, Lal Dangol R. Successful use of tablet personal computers and wireless technologies for the 2011 Nepal Demographic and Health Survey. Glob Health Sci Pract 2013 Jul 11;1(2):277-284. [doi:

10.9745/ghsp-d-12-00056] 
50. Knipe DW, Pearson M, Borgstrøm R, Pieris R, Weerasinghe M, Priyadarshana C, et al. Challenges and opportunities of a paperless baseline survey in Sri Lanka. BMC Res Notes 2014 Jul 15;7(1):-. [doi: 10.1186/1756-0500-7-452]

51. Sime H, Deribe K, Assefa A, Newport MJ, Enquselassie F, Gebretsadik A, et al. Integrated mapping of lymphatic filariasis and podoconiosis: lessons learnt from Ethiopia. Parasit Vectors 2014 Aug 27;7:397 [FREE Full text] [doi: 10.1186/1756-3305-7-397] [Medline: 25164687]

52. Rigby M, Ammenwerth E. The need for evidence in health informatics. Stud Health Technol Inform 2016;222:3-13. [Medline: 27198087]

53. Schweitzer J, Synowiec C. The economics of eHealth and mHealth. J Health Commun 2012 May 02;17 Suppl 1(sup1):73-81. [doi: 10.1080/10810730.2011.649158] [Medline: 22548602]

54. LeFevre AE, Shillcutt SD, Broomhead S, Labrique AB, Jones T. Defining a staged-based process for economic and financial evaluations of mHealth programs. Cost Eff Resour Alloc 2017;15:5 [FREE Full text] [doi: 10.1186/s12962-017-0067-6] [Medline: 28428734]

55. Pham Q, Wiljer D, Cafazzo JA. Beyond the randomized controlled trial: a review of alternatives in mhealth clinical trial methods. JMIR Mhealth Uhealth 2016 Sep 9;4(3):e107 [FREE Full text] [doi: 10.2196/mhealth.5720] [Medline: 27613084]

56. van Poucke S, Thomeer M, Heath J, Vukicevic M. Are randomized controlled trials the (g)old standard? From clinical intelligence to prescriptive analytics. J Med Internet Res 2016 Jul 6;18(7):e185 [FREE Full text] [doi: 10.2196/jmir.5549] [Medline: 27383622]

57. Greenhalgh T, Russell J. Why do evaluations of eHealth programs fail? An alternative set of guiding principles. PLoS Med 2010 Nov 2;7(11):e1000360 [FREE Full text] [doi: 10.1371/journal.pmed.1000360] [Medline: 21072245]

58. Ethiopia Demographic Health Survey 2016. Central Statistical Agency (CSA) [Ethiopia] and ICF. 2016. URL: https:/ /dhsprogram.com/pubs/pdf/FR328/FR328.pdf [accessed 2018-08-12]

59. Edith L. The Effect of Computer-assisted Interviewing on Data Quality: a Review of the Evidence. Utrecht University Repository. 2008. URL: https://dspace.library.uu.nl/handle/1874/44502 [accessed 2020-12-30]

\section{Abbreviations}

CHEERS: Consolidated Health Economic Evaluation Reporting Standards

CRF: clinical case report form

DHS: demographic and health survey

eCRF: electronic clinical report form

HDSS: health and demographic surveillance system

INDEPTH: International Network for the Demographic Evaluation of Populations and Their Health

mHealth: mobile health

RR: risk ratio

Edited by G Eysenbach; submitted 12.06.20; peer-reviewed by R Lundin, M Rauws; comments to author 19.07.20; revised version
received 03.08.20; accepted 28.10.20; published 22.01.21
Please cite as:
Zeleke AA, Naziyok T, Fritz F, Christianson L, Röhrig R
Data Quality and Cost-effectiveness Analyses of Electronic and Paper-Based Interviewer-Administered Public Health Surveys:
Systematic Review
JMed Internet Res 2021;23(1):e21382
URL: $\underline{\text { http://www.jmir.org/2021/1/e21382/ }}$
doi: $\underline{10.2196 / 21382}$
PMID: $\underline{3480859}$

(C)Atinkut Alamirrew Zeleke, Tolga Naziyok, Fleur Fritz, Lara Christianson, Rainer Röhrig. Originally published in the Journal of Medical Internet Research (http://www.jmir.org), 22.01.2021. This is an open-access article distributed under the terms of the Creative Commons Attribution License (https://creativecommons.org/licenses/by/4.0/), which permits unrestricted use, distribution, and reproduction in any medium, provided the original work, first published in the Journal of Medical Internet Research, is properly cited. The complete bibliographic information, a link to the original publication on http://www.jmir.org/, as well as this copyright and license information must be included. 